\title{
Operator limit of the circular beta ensemble
}

\author{
Benedek Valkó and Bálint Virág
}

\begin{abstract}
We provide a precise coupling of the finite circular beta ensembles and their limit process via their operator representations. We prove explicit bounds on the distance of the operators and the corresponding point processes. We also prove an estimate on the beta-dependence in the $\mathrm{Sine}_{\beta}$ process.
\end{abstract}

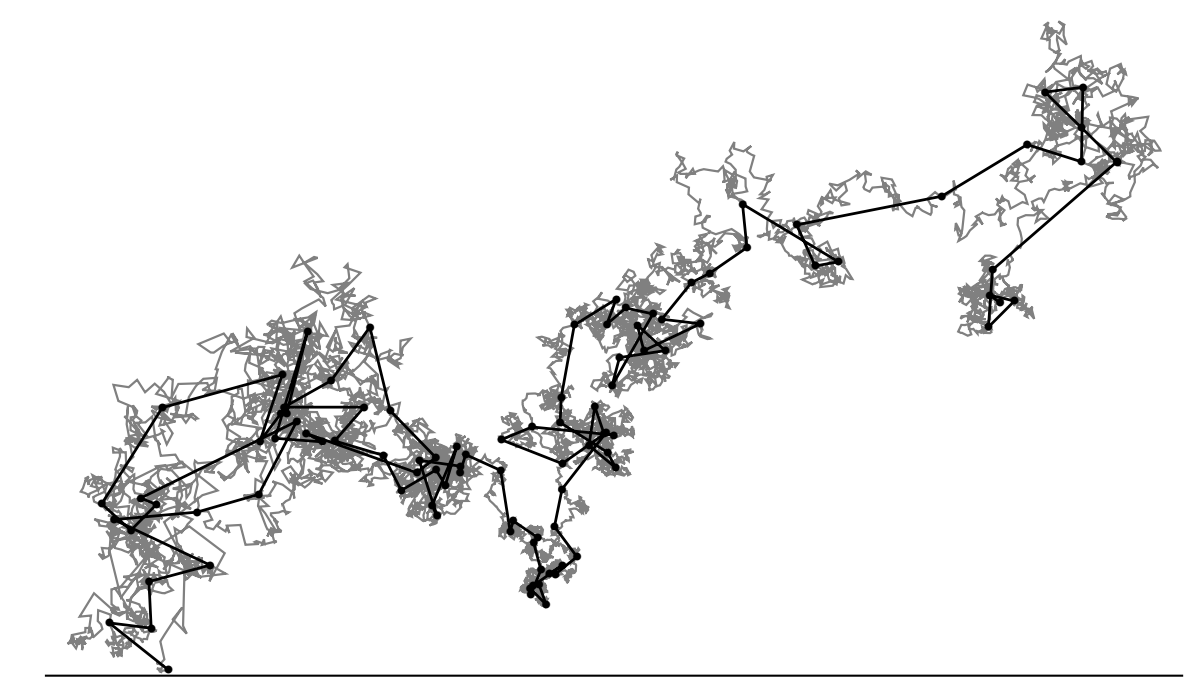

Figure 1: Simulation of hyperbolic Brownian motion and a coupled random walk.

\section{Introduction}

Valkó and Virág (2017) introduced a family of differential operators parametrized by a path $\gamma:[0,1) \rightarrow \mathbb{H}$ in the upper half plane and two points on the boundary.

When the path is a certain hyperbolic random walk in the Poincaré half-plane model, the operator $\operatorname{Circ}_{\beta, n}$ has eigenvalues given by the points of the circular beta ensemble scaled 
and lifted periodically to the real line. With the path $\gamma(t)=\mathcal{B}\left(-\frac{4}{\beta} \log (1-t)\right)$ where $\mathcal{B}$ is standard hyperbolic Brownian motion, the operator $\operatorname{Sine}_{\beta}$ has eigenvalues given by the Sine $_{\beta}$ process, the limit of the circular beta ensembles. (See Theorems 7 and 8 .)

The inverses of these operators in a compatible basis are integral operators denoted by $r \operatorname{Circ}_{\beta, n}$ and $r \operatorname{Sine}_{\beta}$, respectively. Our main result is a coupling which gives $\operatorname{Circ}_{\beta, n} \rightarrow$ Sine $_{\beta}$ with an explicit rate of convergence. (See Section 2 for additional details.)

Theorem 1. There exist an array of stopping times $0=\tau_{n, n}<\tau_{n, n-1}<\cdots<\tau_{n, 0}=\infty$ so that $\mathcal{B}\left(\tau_{n,\lceil(1-t) n\rceil}\right), t \in[0,1)$ has the law of the random walk on $\mathbb{H}$ used to generate $\operatorname{Circ}_{\beta, n}$.

This coupling of $\mathrm{Sine}_{\beta}$ and the sequence of operators $\mathrm{Circ}_{\beta, n}$ satisfies

$$
\left\|r \operatorname{Sine}_{\beta}-r \operatorname{Circ}_{\beta, n}\right\|_{\mathrm{HS}}^{2} \leq \frac{\log ^{6} n}{n}
$$

a.s. in the Hilbert-Schmidt norm for all $n \geq N$, where $N$ is random.

As a corollary, we get new results about the rate of convergence of the eigenvalue processes. Let $\lambda_{k}, k \in \mathbb{Z}$ be the ordered sequence of eigenvalues of $\operatorname{Sine}_{\beta}$ with $\lambda_{0}<0<\lambda_{1}$, the sequence $\lambda_{k, n}, k \in \mathbb{Z}$ is defined analogously for $\operatorname{Circ}_{\beta, n}$.

Corollary 2. In the coupling of Theorem 1 for all $n \geq N$ we have a.s.

$$
\sum_{k \in \mathbb{Z}}\left(\lambda_{k}^{-1}-\lambda_{n, k}^{-1}\right)^{2} \leq \frac{\log ^{6} n}{n} .
$$

Moreover, as $n \rightarrow \infty$ we have a.s.

$$
\max _{|k| \leq \frac{n^{1 / 4}}{\log ^{2} n}}\left|\lambda_{k}-\lambda_{k, n}\right| \rightarrow 0
$$

For all $\varepsilon>0$ there is a random $N_{\varepsilon}$ so that for $n \geq N_{\varepsilon}$ and $|k| \leq n^{1 / 2-\varepsilon}$ we have

$$
\left|\lambda_{k}-\lambda_{k, n}\right| \leq \frac{1+k^{2}}{n^{1 / 2-\varepsilon}}
$$

This provides the best known coupling of the circular $\beta$-ensemble to the $\mathrm{Sine}_{\beta}$ process, even for $\beta=2$, when both processes are determinantal with explicitly given kernels. For $\beta=2$ the bound (4) improves on a coupling given in Maples, Najnudel and Nikeghbali (2013) in which the inequality holds with the exponent $1 / 3$ instead of $1 / 2$, for $|k| \leq n^{1 / 4}$.

Using the techniques introduced in our proof we are also able to give an estimate on the dependence on $\beta$ in the Sine $_{\beta}$ process. 
Theorem 3. Construct the Sine $_{\beta}$ operators for all $\beta>0$ with the same hyperbolic Brownian motion. Denote the eigenvalues corresponding to $\beta$ by $\left\{\lambda_{k, \beta}, k \in \mathbb{Z}\right\}$ with $\lambda_{0, \beta}<0 \leq \lambda_{1, \beta}$. Then for $0<\kappa<1$ there is an a.s. finite $C=C_{\kappa}$ so that if $\kappa<\beta_{1}<\beta<\kappa^{-1}$ and $\delta=\left|\frac{4}{\beta}-\frac{4}{\beta_{1}}\right| \leq 1 / 3$ then

$$
\sum_{k}\left(\frac{1}{\lambda_{k, \beta}}-\frac{1}{\lambda_{k, \beta_{1}}}\right)^{2} \leq\left\|\mathrm{r} \operatorname{Sine}_{\beta}-\mathrm{rine}_{\beta_{1}}\right\|_{\mathrm{HS}} \leq C \delta \log \left(\frac{1}{\delta}\right)
$$

\section{Sructure of the paper}

The proof relies on a precise coupling of a hyperbolic random walk and hyperbolic Brownian motion.

The starting point is a hyperbolic heat kernel bound. Consider the squared Euclidean norm for a hyperbolic Brownian motion started at the origin in the the Poincare disk model. We show that this quantity is very close in total variation to a beta random variable, which also stochastically dominates it (Lemma 9 in Section 3.1).

This leads to a coupling of the hyperbolic random walk steps with hyperbolic Brownian increments at stopping times (Sections 3.2 and 3.3). For each single step, with high probability we stop at a fixed time. Otherwise, we just wait for the Brownian motion to hit the right distance. At the tail of the random walk a slightly different coupling is implemented.

A modulus of continuity estimate for hyperbolic Brownian motion then implies that the random walk is close to the Brownian path (Section 3.4).

In Section 4 we show that if two paths are close and escape linearly to the boundary of $\mathbb{H}$ then the corresponding operators are also close. Finally, in Section 5 we use the the coupling and the linear rate of escape for hyperbolic Brownian motion to prove Theorem 1 .

Section 6 proves Theorem 3 . Some of the technical facts needed are collected in the Appendix.

\section{Historical background}

The modern history of random matrices originates from Wigner (1951), who used them to approximate the spectrum of self-adjoint operators from statistical physics point of view.

In the following decades the scaling behavior of a number of random matrix models were derived. The point process limits of the random matrix spectra were described via the limiting joint densities, usually relying on some algebraic structure of the finite models. (See the monographs Anderson, Guionnet and Zeitouni (2009), Forrester (2010), Mehta (2004) for an overview of the classical results.) 
Dumitriu and Edelman (2002) constructed tridiagonal random matrix models with spectrum distributed as beta ensembles, one parameter extensions of classical random matrix models. Edelman and Sutton (2007) observed that under the appropriate scaling these tridiagonal matrix models behave like approximate versions of random stochastic operators, and conjectured that scaling limits of beta ensembles can be described as the spectra of these objects .

These conjectures were confirmed in Ramírez, Rider and Virág (2011) and Ramírez and Rider (2009) for the the soft and hard edge scaling limits of beta ensembles. The authors rigorously defined the stochastic differential operators that show up as limits, and proved the convergence of the finite ensembles to the spectrum of these operators.

In Valkó and Virág (2009) and Killip and Stoiciu (2009) the bulk scaling limit of the gaussian and circular beta ensembles were derived, and the counting functions of the limit processes were characterized via coupled systems of SDEs. In Nakano (2014) and Valkó and Virág (2017) it was shown that the scaling limit of the circular beta ensemble is the same as $\mathrm{Sine}_{\beta}$, the bulk limit of the gaussian beta ensemble. Furthermore, Valkó and Virág (2017) constructed a stochastic differential operator with a spectrum given by Sine $_{\beta}$.

The coupling of the circular beta ensemble for $\beta=2$ (the circular unitary ensemble) to its limit, the Sine 2 process has been recently studied in Maples et al. (2013) and Meckes and Meckes (2016). In Maples et al. (2013) the circular unitary ensembles of various sizes are coupled together and it is shown that the scaled ensembles converge a.s. to a Sine $_{2}$ process. Moreover, a bound of the form (4) is given with an exponent 1/3 instead of $1 / 2$. In Meckes and Meckes (2016) the total variation distance between the counting functions of the finite and the limiting process is considered. Denote by $\mathcal{N}_{n}$ the counting function of the appropriately scaled circular unitary ensemble of size $n$, and by $\mathcal{N}$ the counting function of the Sine $_{2}$ process. It is shown that for any fixed interval $I$ the following bound holds:

$$
d_{T V}\left(\mathcal{N}_{n}(I), \mathcal{N}(I)\right) \leq 5 \frac{|I|^{2}}{n^{3 / 2}}, \quad \text { for } n \geq n_{0}(I) .
$$

This provides a bound on the distance between the distributions for the number of points in a given interval, but does not seem to imply a process level result.

\section{Stochastic differential operators}

We review the framework introduced in Valkó and Virág (2017) to study random matrix ensembles via differential operators. 


\subsection{Dirac operators}

We consider differential operators of the form

$$
\tau: f \rightarrow R^{-1}(t) J \frac{d}{d t} f
$$

Here $f:[0,1) \rightarrow \mathbb{R}^{2}$, and

$$
J=\left(\begin{array}{cc}
0 & -1 \\
1 & 0
\end{array}\right), \quad R=\frac{1}{2} X^{t} X, \quad X=\frac{1}{\sqrt{y}}\left(\begin{array}{cc}
1 & -x \\
0 & y
\end{array}\right),
$$

with $x:[0,1) \rightarrow \mathbb{R}$ and $y:[0,1) \rightarrow(0, \infty)$. We consider boundary conditions parametrized by nonzero vectors $u_{0}, u_{1} \in \mathbb{R}^{2}$ where we assume that $u_{0}^{t} J u_{1}=1$. We set the domain of the differential operator $\tau$ as

$$
\operatorname{dom}(\tau)=\left\{v \in L_{R}^{2} \cap \mathrm{AC}: \tau v \in L_{R}^{2}, v(0)^{t} J u_{0}=0, \lim _{s \rightarrow 1} v(s)^{t} J u_{1}=0\right\}
$$

Here $L_{R}^{2}$ is the $L^{2}$ space of functions $f:[0,1) \rightarrow \mathbb{R}^{2}$ with the $L^{2}$ norm $\|f\|_{2}^{2}=\int_{0}^{1} f^{t} R f d s$ while AC is the set of absolutely continuous functions.

The function $\gamma=x+i y$ is a path in the upper half plane $\{(x, y): y>0\}$. In Valkó and Virág (2017) it was shown that various properties of $\tau$ can be identified by treating $\gamma$ as a path in the hyperbolic plane $\mathbb{H}$ (using the upper half plane representation) with $u_{0}, u_{1}$ identified with boundary points $\eta_{0}, \eta_{1}$ of $\mathbb{H}$. The set of boundary points of $\mathbb{H}$ in the upper half plane representation is $\mathbb{R} \cup\{\infty\}$. A nonzero $u=\left(u_{1}, u_{2}\right)^{t} \in \mathbb{R}^{2}$ corresponds to the boundary point $\mathcal{P} u=\frac{u_{1}}{u_{2}}$ where this is $\infty$ if $u_{2}=0$. To show the dependence on these parameters, we use the notation $\tau=\operatorname{Dir}\left(\gamma, \eta_{0}, \eta_{1}\right)$.

For a given boundary point $\eta \in \partial \mathbb{H}$ the (signed) horocyclic distance of points $a$ and $b$ in $\mathbb{H}$ with respect to $\eta$ is defined as

$$
d_{\eta}(a, b)=\lim _{z \rightarrow \eta}\left(d_{\mathbb{H}}(a, z)-d_{\mathbb{H}}(b, z)\right)
$$

Here $d_{\mathbb{H}}$ is the hyperbolic distance and the limit is evaluated along a sequence of points in $\mathbb{H}$ converging to $\eta$. We record the following formulas for the half-plane representation:

$$
d_{\eta}(x+i y, i)= \begin{cases}\log \left(\frac{1}{y}\right), & \text { if } \eta=\infty, \\ \log \left(\frac{(x-q)^{2}+y^{2}}{\left(1+q^{2}\right) y}\right), & \text { if } \eta=q \in \mathbb{R} .\end{cases}
$$

The following theorem gives a condition in terms of the parameters $\gamma, \eta_{0}, \eta_{1}$ for $\tau$ to be self-adjoint with a Hilbert-Schmidt inverse. 
Theorem 4 (Valkó and Virág (2017)). Let $\eta_{0}, \eta_{1}$ be distinct boundary points of $\mathbb{H}$ and $\gamma:[0,1) \rightarrow \mathbb{H}$ be measurable and locally bounded. Assume that there is a $\xi \in \mathbb{H}$ with

$$
\int_{0}^{T} e^{d_{\eta_{1}}(\gamma(t), \xi)} d t<\infty, \quad \text { and } \quad \int_{0}^{T} \int_{s}^{T} e^{d_{\eta_{0}}(\gamma(s), \xi)+d_{\eta_{1}}(\gamma(s), \xi)} d s d t<\infty .
$$

Then the operator $\tau=\operatorname{Dir}\left(\gamma, \eta_{0}, \eta_{1}\right)$ is self-adjoint on $\operatorname{dom}(\tau)$ and its inverse is HilbertSchmidt. The inverse $\tau^{-1}$ is an integral operator on $L_{R}^{2}$ with kernel function

$$
K(x, y)=\left(u_{0} u_{1}^{t} \mathbf{1}(x<y)+u_{1} u_{0}^{t} \mathbf{1}(x \geq y)\right) R(y) .
$$

Suppose that $\gamma, \eta_{0}, \eta_{1}$ satisfy the conditions of the theorem above and consider the operator $\tau=\operatorname{Dir}\left(\gamma, \eta_{0}, \eta_{1}\right)$. Let $\hat{\tau}=X \tau X^{-1}$, this is just $\tau$ after a change of coordinates. In particular $\hat{\tau}$ is a self-adjoint differential operator on $\{X v \in \operatorname{dom}(\tau)\} \subset L^{2}$, with the same spectrum as $\tau$. We denote the inverse of $\hat{\tau}$ by $\mathrm{r} \tau$ ( $\mathrm{r}$ standing for resolvent). By Theorem 4 the operator $r \tau$ is an integral operator acting on $L^{2}$ functions with kernel

$$
K_{\mathrm{r} \tau}(x, y)=\frac{1}{2}\left(a(x) c(y)^{t} \mathbf{1}(x<y)+c(x) a(y)^{t} \mathbf{1}(x \geq y)\right)
$$

where $a(s)=X(s) u_{0}$ and $c(s)=X(s) u_{1}$.

\section{$2.2 \quad$ Stochastic operators}

The gaussian and circular $\beta$ ensembles are defined via the following joint densities on $\mathbb{R}^{n}$ and $[0,2 \pi)^{n}$ :

$$
\begin{aligned}
& p_{\beta, n}^{\mathrm{g}}\left(\lambda_{1}, \ldots, \lambda_{n}\right)=\frac{1}{Z_{n, \beta}^{\mathrm{g}}} \prod_{1 \leq j<k \leq n}\left|\lambda_{j}-\lambda_{k}\right|^{\beta} e^{-\frac{\beta}{4} \sum_{j=1}^{n} \lambda_{j}^{2}}, \\
& p_{\beta, n}^{\mathrm{c}}\left(\lambda_{1}, \ldots, \lambda_{n}\right)=\frac{1}{Z_{n, \beta}^{\mathrm{c}}} \prod_{1 \leq j<k \leq n}\left|e^{i \lambda_{j}}-e^{i \lambda_{k}}\right|^{\beta} .
\end{aligned}
$$

For $\beta=2$ these give the joint eigenvalue densities of the gaussian and circular unitary ensemble, respectively. We use angles to represent the eigenvalues in the circular case.

The bulk scaling limits of these ensembles have been identified in Valkó and Virág (2009) and Killip and Stoiciu (2009). In Nakano (2014) and Valkó and Virág (2017) it was shown that the scaling limit of the circular beta ensemble is the same as the bulk limit of the gaussian beta ensemble.

Theorem 5 (Valkó and Virág (2009)). Fix $\beta>0$ and $|E|<2$. Let $\Lambda_{n}^{\mathrm{g}}$ be a finite point process with density 14 . Then $\sqrt{4-E^{2}} \sqrt{n}\left(\Lambda_{n}^{g}-\sqrt{n} E\right)$ converges in distribution to a point process Sine $_{\beta}$. 
Theorem 6 (Killip and Stoiciu (2009), Nakano (2014), Valkó and Virág (2017)). Fix $\beta>0$ and let $\Lambda_{n}^{\mathrm{c}}$ be a finite point process with density (15). Then $n \Lambda_{n}^{c}$ converges in distribution to the point process Sine $_{\beta}$.

In Valkó and Virág (2017) the authors constructed random Dirac operators with spectrum given by Sine $_{\beta}$ and the finite circular beta ensemble. Recall that the standard hyperbolic Brownian motion $x+i y$ in the upper half plane representation started from $i$ is the solution of the SDE

$$
d(x+i y)=y\left(d B_{1}+i d B_{2}\right), \quad x(0)+i y(0)=i,
$$

where $B_{1}, B_{2}$ are independent standard real Brownian motions.

Theorem 7 (Valkó and Virág (2017)). Fix $\beta>0$ and let $\mathcal{B}$ be a standard hyperbolic Brownian motion in the upper half plane started from $\mathcal{B}(0)=i$. Set $\tilde{\mathcal{B}}(t)=\mathcal{B}\left(-\frac{4}{\beta} \log (1-t)\right), t \in$ $[0,1), \eta_{0}=\infty$ and $\eta_{1}=\lim _{t \rightarrow \infty} \mathcal{B}(t)$. Then the operator

$$
\operatorname{Sine}_{\beta}=\operatorname{Dir}\left(\tilde{\mathcal{B}}(t), \eta_{0}, \eta_{1}\right)
$$

is a.s. self-adjoint with a Hilbert-Schmidt inverse and $\operatorname{spec}\left(\operatorname{Sine}_{\beta}\right) \stackrel{d}{=} \operatorname{Sine}_{\beta}$.

The random Dirac operator for the finite circular beta ensemble is defined using a random walk in $\mathbb{H}$. Fix $n \geq 1$ and let $\zeta_{0}, \ldots, \zeta_{n-2}$ be independent with $\zeta_{k} \sim \operatorname{Beta}\left(1, \frac{\beta}{2}(n-k-1)\right)$. 1 Set $Y_{k}=\log \left(\frac{1+\sqrt{\zeta_{k}}}{1-\sqrt{\zeta_{k}}}\right)$. We define the random walk $b_{0}, b_{1}, \ldots, b_{n-1}$ in $\mathbb{H}$ with a final boundary point $b_{n} \in \partial \mathbb{H}$. We set $b_{0}=i$, and for $0 \leq k \leq n-2$ we choose $b_{k+1}$ uniformly among the points in $\mathbb{H}$ with hyperbolic distance $Y_{k}$ from $b_{k}$, independently of the previous choices. The final point $b_{n}$ is chosen uniformly on the boundary $\partial \mathbb{H}$ as viewed from $b_{n-1}$, independently of the previous choices. Note that $\zeta_{k} \sim \operatorname{Beta}\left(1, \frac{\beta}{2}(n-k-1)\right)$ is the squared Euclidean norm of the random walk step in the Poincare disk model with $b_{k}$ at the origin.

Theorem 8 (Valkó and Virág (2017)). Consider the random walk $b_{0}, b_{1}, \ldots, b_{n}$ defined above. Set $\eta_{0}=\infty, \eta_{1}=b_{n}$ and $\mathcal{B}_{n}(t)=b_{\lfloor n t\rfloor}$ for $t \in[0,1)$. The operator

$$
\operatorname{Circ}_{\beta, n}=\operatorname{Dir}\left(\mathcal{B}_{n}(t), \eta_{0}, \eta_{1}\right)
$$

is a.s. self-adjoint with a Hilbert-Schmidt inverse and $\operatorname{spec}\left(\operatorname{Circ}_{\beta, n}\right) \stackrel{d}{=} n \Lambda_{n}^{\mathrm{c}}+2 \pi \mathbb{Z}$ where $\Lambda_{n}^{\mathrm{c}}$ is the finite point process with density (15).

Theorems 7 and 8 imply that almost surely zero is not an eigenvalue for the operators Sine $_{\beta}$ and $\operatorname{Circ}_{\beta, n}$.

\footnotetext{
${ }^{1}$ A random variable has distribution $\operatorname{Beta}(a, b)$ with $a, b>0$ if it has density $\frac{x^{a-1}(1-x)^{b-1}}{\Gamma(a) \Gamma(b)} \mathbf{1}(x \in[0,1])$.
} 


\subsection{Coupling $\operatorname{Circ}_{\beta, n}$ and Sine $\beta$}

Fix $\beta>0$. Let $\mathcal{B}$ a hyperbolic Brownian motion, set $\eta_{0}=\infty, \eta_{1}=\mathcal{B}(\infty)$ and Sine $_{\beta}=$ $\operatorname{Dir}\left(\mathcal{B}\left(-\frac{4}{\beta} \log (1-t)\right), \eta_{0}, \eta_{1}\right)$.

Let $U$ be a uniform random variable on $[0,1]$ independent from $\mathcal{B}$, and let $\mathcal{F}_{t}, t \geq 0$ be the natural filtration of $\mathcal{B}$ enlarged with $U$. Theorem 1 gives the existence of an array of stopping times $\tau_{n, k}$ with respect to $\mathcal{F}_{t}$ so that for each $n \geq 1$ the random variables $\mathcal{B}\left(\tau_{n, n}\right), \mathcal{B}\left(\tau_{n, n-1}\right), \ldots, \mathcal{B}\left(\tau_{n, 0}\right)$ have the same joint distribution as the random walk $b_{0}, \ldots, b_{n}$ from Theorem 8. Setting $\operatorname{Circ}_{\beta, n}=\operatorname{Dir}\left(\tilde{\mathcal{B}}_{n}(t), \eta_{0}, \eta_{1}\right)$ with $\tilde{\mathcal{B}}_{n}(t)=\mathcal{B}\left(\tau_{n,\lceil(1-t) n]}\right), t \in[0,1)$ gives the appropriate coupling of $\operatorname{Sine}_{\beta}$ and the sequence $\left\{\operatorname{Circ}_{\beta, n}\right\}_{n \geq 1}$.

\section{Coupling construction}

\subsection{A heat kernel bound on the hyperbolic plane}

Our coupling relies on a careful estimate of the transition density for hyperbolic Brownian motion. Although there are a number of existing bounds in the literature (see e.g. Davies and Mandouvalos (1988)), we could not find one that would be strong enough for our purposes. We show that the distribution of the distance of hyperbolic Brownian motion at time $t \leq 1$ from its starting point can be very precisely estimated in terms of a Beta $\left(1, \frac{2}{t}-1 / 2\right)$ random variable.

Lemma 9. Let $\mathcal{B}(t)$ be standard hyperbolic Brownian motion and let $t \in(0,1]$. Let $Y=$ $\log \left(\frac{1+\sqrt{\xi}}{1-\sqrt{\xi}}\right)$ where $\xi$ has distribution $\operatorname{Beta}\left(1, \frac{2}{t}-1 / 2\right)$ and set $\zeta=d_{\mathbb{H}}(\mathcal{B}(0), \mathcal{B}(t))$. Then the following statements hold:

(a) $P(Y>r) \geq P(\zeta>r)$ for all $r>0$, in other words $Y$ stochastically dominates $\zeta$.

(b) The total variation distance of $\zeta$ and $Y$ is bounded by $\frac{3}{2} t$.

The proof of the lemma relies on a precise analysis of the explicit formula for the transition density. We leave it for Section 7.1 in the Appendix.

\subsection{Single step coupling}

We first concentrate on a single step in the hyperbolic random walk corresponding to $\operatorname{Circ}_{\beta, n}$ and couple it to the hyperbolic Brownian motion. 
Proposition 10. Fix $\gamma \geq 3 / 2$. Let $\mathcal{B}$ be hyperbolic Brownian motion and $U$ an independent uniform random variable on $[0,1]$. Let $\mathcal{F}_{t}, t \geq 0$ be the filtration of $\mathcal{B}$ enlarged with $U$. Consider a Poincaré disk representation of the hyperbolic plane where $\mathcal{B}(0)=0$.

There exists a finite random variable $\sigma>0$ so that the following hold:

1. $\sigma$ is a stopping time with respect to $\mathcal{F}_{t}$.

2. $\mathcal{B}(\sigma)$ has rotationally invariant distribution and $|\mathcal{B}(\sigma)|^{2}$ has $\operatorname{Beta}(1, \gamma)$ distribution.

3. $P\left(\sigma \geq \frac{4}{2 \gamma+1}\right)=1$ and $P\left(\sigma \neq \frac{4}{2 \gamma+1}\right) \leq \frac{3}{\gamma}$.

4. For $r \geq 8$ we have $P(\sigma>r / \gamma) \leq 3 e^{-\frac{1}{5} r^{1 / 3}}$.

The proof of the proposition will rely on Lemma 9 and the lemma below, to be proved in the Appendix.

Lemma 11. Assume that $X_{1}$ and $X_{2}$ are random variables so that $X_{2}$ stochastically dominates $X_{1}$ and the total variation distance of their distributions is $\varepsilon$. Then there exists a measurable function $g: \mathbb{R}^{2} \rightarrow \mathbb{R}$ so that if $U$ is a uniform random variable on $[0,1]$, independent of $X_{1}$ then the following hold:

(a) $g\left(X_{1}, U\right)$ has the same distribution as $X_{2}$.

(b) $P\left(X_{1} \leq g\left(X_{1}, U\right)\right)=1$.

(c) $P\left(X_{1}=g\left(X_{1}, U\right)\right)=1-\varepsilon$.

Proof of Proposition 10. Set $t=\frac{4}{2 \gamma+1}$, then $0<t<1$. Recall that if $z$ is in the Poincaré disk with $|z|=r<1$ then $d_{\mathbb{H}}(0, z)=\log \left(\frac{1+r}{1-r}\right)$.

Let $\xi$ be a random variable with distribution $\operatorname{Beta}(1, \gamma)$. Then by Lemma 9 the random variable $\log \left(\frac{1+|\mathcal{B}(t)|}{1-|\mathcal{B}(t)|}\right)$ is stochastically dominated by $\log \left(\frac{1+\sqrt{\xi}}{1-\sqrt{\xi}}\right)$ and their total variation distance is bounded by $\frac{3}{2} t$. Since $\log \left(\frac{1+r}{1-r}\right)$ is strictly increasing in $r$, we get that $|\mathcal{B}(t)|$ is stochastically dominated by $\sqrt{\xi}$ and their total variation distance is bounded by $\frac{3}{2} t$.

By Lemma 11 there exits a measurable function $g$ so that $g(|\mathcal{B}(t)|, U) \geq|\mathcal{B}(t)|$ almost surely, $g(|\mathcal{B}(t)|, U)$ has the same distribution as $\sqrt{\xi}$, and $P(|\mathcal{B}(t)| \neq g(|\mathcal{B}(t)|, U)) \leq \frac{3}{2} t \leq 3 / \gamma$.

We set

$$
\sigma=\inf \{s \geq t:|\mathcal{B}(s)|=g(|\mathcal{B}(t)|, U)\} .
$$

Then $\sigma$ is an a.s. finite stopping time with respect to $\mathcal{F}_{t}$ and almost surely $\sigma \geq t$. Because $\sigma$ only depends on $|\mathcal{B}|$ and $U$, it follows that $\mathcal{B}(\sigma)$ has rotationally invariant distribution. Finally, from our construction we get that $|\mathcal{B}(\sigma)|^{2}=g(|\mathcal{B}(t)|, U)^{2}$ has Beta $(1, \gamma)$ distribution and $P(\sigma \neq t) \leq \frac{3}{2} t \leq 3 / \gamma$. 
The only thing left to prove is the tail bound for $\sigma$. We start with the bound

$$
P(\sigma>r / \gamma) \leq P\left(\sigma>r / \gamma \text { and } d_{\mathbb{H}}(0, \mathcal{B}(\sigma)) \leq \frac{r^{1 / 3}}{\gamma^{1 / 2}}\right)+P\left(d_{\mathbb{H}}(0, \mathcal{B}(\sigma))>\frac{r^{1 / 3}}{\gamma^{1 / 2}}\right)
$$

For the rest of the proof we assume $r \geq 8$. Then $\sigma>r / \gamma>t$ and from the definition of $\sigma$ it follows that $d_{\mathbb{H}}(0, \mathcal{B}(s))<d_{\mathbb{H}}(0, \mathcal{B}(\sigma))$ for $t \leq s<\sigma$. Thus we can bound the first term on the right of 16 by writing

$$
\begin{aligned}
P\left(\sigma>r / \gamma \text { and } d_{\mathbb{H}}(0, \mathcal{B}(\sigma)) \leq \frac{r^{1 / 3}}{\gamma^{1 / 2}}\right) & \leq P\left(\max _{t \leq s \leq r / \gamma} d_{\mathbb{H}}(0, \mathcal{B}(s)) \leq \frac{r^{1 / 3}}{\gamma^{1 / 2}}\right) \\
& \leq P\left(\max _{t \leq s \leq r / \gamma} d_{\mathbb{H}}(\mathcal{B}(t), \mathcal{B}(s)) \leq 2 \frac{r^{1 / 3}}{\gamma^{1 / 2}}\right) \\
& =P\left(\max _{0 \leq s \leq r / \gamma-t} d_{\mathbb{H}}(0, \mathcal{B}(s)) \leq 2 \frac{r^{1 / 3}}{\gamma^{1 / 2}}\right)
\end{aligned}
$$

Since $t \leq \frac{3}{2 \gamma}$ and $r \geq 8$, we have $r / \gamma-t \geq \frac{4}{5} r \gamma^{-1}$. Using the bound (52) from Lemma 20 of the Appendix we get

$$
P\left(\max _{0 \leq s \leq r / \gamma-t} d_{\mathbb{H}}(0, \mathcal{B}(s)) \leq 2 \frac{r^{1 / 3}}{\gamma^{1 / 2}}\right) \leq P\left(\max _{0 \leq s \leq \frac{4}{5} r \gamma^{-1}} d_{\mathbb{H}}(0, \mathcal{B}(s)) \leq 2 \frac{r^{1 / 3}}{\gamma^{1 / 2}}\right) \leq \frac{4}{\pi} e^{-\frac{\pi^{2} r^{1 / 3}}{40}} .
$$

For the second term in $(16)$ we recall that by construction $d_{\mathbb{H}}(0, \mathcal{B}(\sigma))$ has the same distribution as $\log \left(\frac{1+\sqrt{\xi}}{1-\sqrt{\xi}}\right)$ where $\xi$ has distribution $\operatorname{Beta}(1, \gamma)$. By an explicit computation

$$
P\left(\log \left(\frac{1+\sqrt{\xi}}{1-\sqrt{\xi}}\right)>u\right)=\operatorname{sech}^{2 \gamma}\left(\frac{u}{2}\right) .
$$

We have $\operatorname{sech}(x) \leq 2 e^{-x}$ which gives the following upper bound for $u \geq 4 \log 2$ :

$$
P\left(\log \left(\frac{1+\sqrt{\xi}}{1-\sqrt{\xi}}\right)>u\right) \leq 2^{2 \gamma} e^{-\gamma u} \leq e^{-\frac{\gamma u}{2}}
$$

For $0 \leq u \leq 4 \log 2$ we have $\log \operatorname{sech}(u / 2) \leq-\frac{u^{2}}{12}$ so for these values we get

$$
P\left(\log \left(\frac{1+\sqrt{\xi}}{1-\sqrt{\xi}}\right)>u\right) \leq e^{-\frac{\gamma u^{2}}{6}}
$$

From this we get

$$
P\left(d_{\mathbb{H}}(0, \mathcal{B}(\sigma))>\frac{r^{1 / 3}}{\gamma^{1 / 2}}\right) \leq \max \left(e^{-\frac{\sqrt{\gamma} r^{1 / 3}}{2}}, e^{-\frac{r^{2 / 3}}{6}}\right) \leq e^{-\frac{1}{3} r^{1 / 3}}
$$

where in the last step we used $\gamma \geq 3 / 2$ and $r \geq 8$. Collecting our estimates we get

$$
P(\sigma>r / \gamma) \leq \frac{4}{\pi} e^{-\frac{\pi^{2} r^{1 / 3}}{40}}+e^{-\frac{1}{3} r^{1 / 3}} \leq 3 e^{-\frac{1}{5} r^{1 / 3}}
$$

which completes the proof of the proposition. 


\subsection{Path coupling}

Proposition 12. Let $\mathcal{B}$ be hyperbolic Brownian motion and $U$ an independent Uniform $[0,1]$ random variable. Let $\mathcal{F}_{t}, t \geq 0$ be the filtration of $\mathcal{B}$ enlarged with $U$.

There exists a collection of stopping times $\left(\tau_{n, k} ; 1 \leq n, 0 \leq k \leq n\right)$ with respect to $\mathcal{F}_{t}, t \geq 0$ so that the following statements hold.

1. For each fixed $n$ we have $0=\tau_{n, n}<\tau_{n, n-1} \ldots<\tau_{n, 0}=\infty$, and $\Delta \tau_{n, k}:=\tau_{n, k}-\tau_{n, k+1}$ are independent.

2. For each $n$, the process $\left(b_{k}^{(n)}=\mathcal{B}\left(\tau_{n, n-k}\right), k=0,1, \ldots, n\right)$ is a hyperbolic random walk with the same distribution as the one given before Theorem 8 .

3. Let $t_{n, k}=\frac{4}{\beta} \log \left(\frac{n}{k}\right)$ for $1 \leq k \leq n$. There exists a random integer $N_{0}>0$ so that for $n \geq N_{0}$ and $\log ^{6} n \leq k \leq n$ we have almost surely

$$
t_{n, k}-\frac{4}{\beta^{2} k} \leq \tau_{n, k} \leq t_{n, k}+\frac{\log ^{4+1 / 2} n}{k} .
$$

4. For $k$ fixed the hyperbolic distance $d_{\mathbb{H}}\left(\mathcal{B}\left(\tau_{n, k}\right), \mathcal{B}\left(\tau_{n, k-1}\right)\right)$ does not depend on $n$ as long as $k<\log ^{6} n$.

Proof. Using a standard argument we can find measurable functions $f_{1, k}, f_{2, k}$ so that $U_{k}=$ $f_{1, k}(U)$ is a Uniform $[0,1]$ random variable, $\xi_{k}=f_{2, k}(U)$ is distributed as $\operatorname{Beta}\left(1, \frac{\beta}{2} k\right)$ and $U_{1}, U_{2}, \ldots, \xi_{1}, \xi_{2}, \ldots$ are independent.

We first give the construction of the stopping times, then prove that they satisfy all the conditions.

For a fixed $n \geq 1$ we define $\tau_{n, k}$ recursively, starting with $\tau_{n, n}=0$. If for a certain $k<n$ we have already defined $\tau_{n, k+1}$ then we define $\tau_{n, k}$ as follows.

- In the case of $k \geq \max \left(\log ^{6} n, \frac{3}{\beta}\right)$ :

We apply Proposition 10 with $\gamma=\frac{\beta}{2} k$ for the hyperbolic Brownian motion $\tilde{\mathcal{B}}(t)=$ $\mathcal{B}\left(t+\tau_{n, k+1}\right), t \geq 0$ and the independent uniform random variable $U_{k}$, and denote the constructed stopping time by $\sigma_{n, k}$. We set $\tau_{n, k}=\tau_{n, k+1}+\sigma_{n, k}$.

- In the case of $1 \leq k<\max \left(\log ^{6} n, \frac{3}{\beta}\right)$ :

We set

$$
\tau_{n, k}=\inf \left\{t \geq \tau_{n, k+1}: d_{\mathbb{H}}\left(\mathcal{B}\left(\tau_{n, k+1}\right), \mathcal{B}(t)\right)=\log \left(\frac{1+\sqrt{\xi_{k}}}{1-\sqrt{\xi_{k}}}\right)\right\}
$$

- For $k=0$ we define $\tau_{n, k}=\infty$. 
By construction, the random variables $\tau_{n, k}$ are a.s. finite stopping times with respect to the filtration $\mathcal{F}_{t}, t \geq 0$, and they satisfy conditions 1,2 and 4 . To check Condition 3 we first choose $n_{0}$ so that for $n \geq n_{0}$ we have $\log ^{6} n \geq \frac{3}{\beta}$ and $\log ^{3+3 / 8} n \geq 8$. During the rest of this proof we will assume $1 \leq \log ^{6} n \leq k \leq n$.

For $n \geq n_{0}$, by Proposition 10 we have almost surely

$$
\tau_{n, k} \geq \sum_{j=k}^{n-1} \frac{4}{\beta j+1} \geq \frac{4}{\beta} \log \left(\frac{n}{k}\right)-\frac{4}{\beta^{2}} \frac{1}{k} .
$$

This takes care of the lower bound in (17).

For the upper bound recall the definition of $\sigma_{n, k}=\tau_{n, k}-\tau_{n, k+1}$. From Proposition 10 we have the following estimates:

$$
\begin{aligned}
& P\left(\sigma_{n, k} \geq \frac{4}{\beta k+1}\right)=1, \quad P\left(\sigma_{n, k}=\frac{4}{\beta k+1}\right) \geq 1-\frac{6}{\beta k}, \\
& P\left(\sigma_{n, k}>\frac{\log ^{3+3 / 8} n}{\frac{\beta}{2} k}\right) \leq 3 e^{-\frac{1}{5} \log ^{1+1 / 8} n} .
\end{aligned}
$$

Since $\sum_{k, n} P\left(\sigma_{n, k}>\frac{\log ^{3+3 / 8} n}{\frac{\beta}{2} k}\right) \leq 3 \sum_{n} n e^{-\frac{1}{3} \log ^{1+1 / 8} n}<\infty$, by the Borel-Cantelli lemma there is a random $N_{1} \geq n_{0}$ so that for $n \geq N_{1}$ we have $\sigma_{n, k} \leq \frac{\log ^{3+3 / 8} n}{\frac{\beta}{2} k}$ a.s. Set

$$
Z_{n, k}=\frac{4}{\beta k+1} \mathbf{1}\left(\sigma_{n, k}=\frac{4}{\beta k+1}\right)+\frac{2 \log ^{3+3 / 8} n}{\beta k} \mathbf{1}\left(\sigma_{n, k} \neq \frac{4}{\beta k+1}\right) .
$$

For $n \geq N_{1}$ we have $\sigma_{n, k} \leq Z_{n, k}$ and $\tau_{n, k} \leq \sum_{j=k}^{n-1} Z_{n, j}$ a.s.

Next we will bound $P\left(\sum_{j=k}^{n-1}\left(Z_{n, j}-\frac{4}{\beta j+1}\right) \geq \frac{\log ^{9 / 2} n}{k}\right)$. For $\lambda>0$ from 18 and 19 we get

$$
E\left(e^{\sum_{j=k}^{n-1} \lambda\left(Z_{n, j}-\frac{4}{\beta k+1}\right)}\right) \leq \prod_{j=k}^{n-1}\left(1-\frac{6}{\beta j}+\frac{6}{\beta j} e^{\lambda \frac{2 \log ^{3+3 / 8} n}{\beta j}}\right) .
$$

Assuming that $\lambda \frac{2 \log ^{3+3 / 8} n}{\beta k} \leq 1$ we can use that $\frac{e^{x}-1}{x} \leq 2$ for $x \leq 1$ to bound the right side of (20) as

$$
\begin{aligned}
\prod_{j=k}^{n-1}\left(1-\frac{6}{\beta j}+\frac{6}{\beta j} e^{\lambda \frac{2 \log ^{3+3 / 8} n}{\beta j}}\right) & \leq \prod_{j=k}^{n-1}\left(1+\frac{6}{\beta j} \cdot 2 \lambda \frac{2 \log ^{3+3 / 8} n}{\beta j}\right) \leq \prod_{j=k}^{n-1} e^{\frac{24 \lambda \log ^{3+3 / 8} n}{\beta^{2} j^{2}}} \\
& \leq e^{\frac{48 \lambda \log ^{3+3 / 8} n}{\beta^{2} k}} .
\end{aligned}
$$

Setting now $\lambda=\frac{\beta k}{2 \log ^{3+3 / 8} n}$ and using the exponential Markov inequality we obtain

$$
\begin{aligned}
P\left(\sum_{j=k}^{n-1}\left(Z_{n, j}-\frac{4}{\beta j+1}\right) \geq \frac{\log ^{4+1 / 2} n}{k}\right) & =E\left(e^{\sum_{j=k}^{n-1} \lambda\left(Z_{n, j}-\frac{4}{\beta k+1}\right)}\right) e^{-\lambda \frac{\log ^{4+1 / 2} n}{k}} \\
& \leq e^{\frac{48 \lambda \log ^{3+3 / 8} n}{\beta^{2} k}-\lambda \frac{\log ^{4+1 / 2} n}{k}} \leq e^{\frac{24}{\beta}-\frac{\beta}{2} \log ^{1+1 / 8} n} .
\end{aligned}
$$


Since $\sum_{n} n e^{\frac{24}{\beta}-\frac{\beta}{2} \log ^{1+1 / 8} n}<\infty$, the Borel-Cantelli lemma implies that there is a random $N_{0} \geq N_{1}$ so that for $n \geq N_{0}$ and $\log ^{6} n \leq k \leq n$ we have a.s.

$$
\sum_{j=k}^{n-1} Z_{n, j} \leq \sum_{j=k}^{n-1} \frac{4}{\beta j+1}+\frac{\log ^{4+1 / 2} n}{k} \leq \frac{4}{\beta} \log \left(\frac{n}{k}\right)+\frac{\log ^{4+1 / 2} n}{k} .
$$

Since $\tau_{n, k} \leq \sum_{j=k}^{n-1}$, the upper bound in 17 follows.

\subsection{Path comparison}

Let $\mathcal{B}$ be a hyperbolic Brownian motion and consider the stopping times $\tau_{n, k}$ constructed in Proposition 12. Set $\tilde{\mathcal{B}}_{n}(t)=\mathcal{B}\left(\tau_{n,\lceil(1-t) n\rceil}\right)$ and $\tilde{\mathcal{B}}(t)=\mathcal{B}\left(-\frac{4}{\beta} \log (1-t)\right)$. The next proposition gives bounds on $d_{\mathbb{H}}\left(\tilde{\mathcal{B}}(t), \tilde{\mathcal{B}}_{n}(t)\right)$.

Proposition 13. There is a random integer $N^{*}$ so that for $n \geq N^{*}$ we have the following a.s. inequalities:

$$
\begin{gathered}
d_{\mathbb{H}}\left(\tilde{\mathcal{B}}(t), \tilde{\mathcal{B}}_{n}(t)\right) \leq \frac{\log ^{3-1 / 8} n}{\sqrt{(1-t) n}}, \quad \text { if } \quad 0 \leq t \leq 1-\frac{\log ^{6} n}{n}, \\
d_{\mathbb{H}}\left(\tilde{\mathcal{B}}_{n}\left(1-\frac{\log ^{6} n}{n}\right), \tilde{\mathcal{B}}_{n}(t)\right) \leq \frac{144}{\beta}(\log \log n)^{2}, \quad \text { if } \quad 1-\frac{\log ^{6} n}{n} \leq t<1 .
\end{gathered}
$$

Proof. Let $T_{n}=1-\frac{\log ^{6} n}{n}$. Consider $N_{0}$ from the statement of Proposition 12. For $n \geq N_{0}$ and $0 \leq t \leq T_{n}$ we have a.s.

$$
\left|\tau_{n,\lceil(1-t) n\rceil}-\frac{4}{\beta} \log \left(\frac{1}{1-t}\right)\right| \leq \frac{4}{\beta^{2}\lceil(1-t) n\rceil}+\frac{\log ^{9 / 2} n}{\lceil(1-t) n\rceil}+\frac{4}{\beta}\left|\log \left(\frac{n}{\lceil(1-t) n\rceil}\right)-\log \left(\frac{1}{1-t}\right)\right| \leq \frac{\log ^{4+5 / 8} n}{(1-t) n},
$$

where for the second inequality we also assume $n \geq n_{0}$ with an $n_{0}$ only depending on $\beta$. Inequality 23 implies

$$
d_{\mathbb{H}}\left(\tilde{\mathcal{B}}(t), \tilde{\mathcal{B}}_{n}(t)\right) \leq \max \left\{d_{\mathbb{H}}(\mathcal{B}(s), \mathcal{B}(s+u)):|u| \leq h, 0 \leq s+u\right\},
$$

with $h=\frac{\log ^{4+5 / 8} n}{(1-t) n}$ and $s=\frac{4}{\beta} \log \left(\frac{1}{1-t}\right)$. Note that $h \leq \frac{\log ^{4+5 / 8} n}{\log ^{6} n} \leq \frac{1}{\log n}$.

Consider the random constant $h_{0}$ from the statement of Proposition 22 of the Appendix. If $n \geq e^{h_{0}^{-1}}$ then $h \leq h_{0}$ and we may apply Proposition 22 with $s, s+u$ if $0 \leq u \leq h$ and with $s+u, s$ if $0 \leq-u \leq \min (h, s)$. Using the fact that $h \log \left(2+\frac{s+1}{h}\right)$ is monotone increasing in $h$ and $s$ we get

$$
\max \left\{d_{\mathbb{H}}(\mathcal{B}(s), \mathcal{B}(s+u)):|u| \leq h, 0 \leq s+u\right\} \leq 20 \sqrt{h \log \left(2+\frac{s+1}{h}\right)} \leq \frac{\log ^{3-1 / 8} n}{\sqrt{(1-t) n}}
$$


if $0 \leq t \leq T_{n}$ and $n \geq N_{1}$, with a random integer $N_{1}$.

To get the estimate for the $1-T_{n} \leq t<1$ case we use the construction given in the proof of Proposition 12 and the triangle inequality repeatedly to get

$$
d_{\mathbb{H}}\left(\tilde{\mathcal{B}}_{n}\left(T_{n}\right), \tilde{\mathcal{B}}_{n}(t)\right) \leq \sum_{j=1}^{\log ^{6} n-1} d_{\mathbb{H}}\left(\mathcal{B}_{n}\left(\frac{n-j}{n}\right), \mathcal{B}_{n}\left(\frac{n-j-1}{n}\right)\right)=\sum_{j=1}^{\log ^{6} n-1} Y_{j}
$$

Here $Y_{j}=\log \left(\frac{1+\sqrt{\xi_{j}}}{1-\sqrt{\xi_{j}}}\right)$ and $\xi_{j} \sim \operatorname{Beta}\left(1, \frac{\beta}{2} j\right)$ are independent random variables. We have

$$
P\left(Y_{j} \geq r\right)=\operatorname{sech}^{\beta j}(r / 2) \leq 2 e^{-\frac{\beta}{2} j r}
$$

which implies $\sum_{j} P\left(Y_{j} \geq \frac{4}{\beta} \frac{\log j}{j}\right)<\infty$. This means that there is a random $M_{0}$ so that for $m \geq M_{0}$ we have $Y_{m} \leq \frac{4}{\beta} \frac{\log m}{m}$ and also $\sum_{j=1}^{m} Y_{j} \leq \frac{4}{\beta} \log ^{2} m$. Thus if $\log ^{6} n \geq M_{0}$ then for $1-T_{n} \leq t<1$ we have almost surely

$$
d_{\mathbb{H}}\left(\tilde{\mathcal{B}}_{n}\left(T_{n}\right), \tilde{\mathcal{B}}_{n}(t)\right) \leq \frac{144}{\beta}(\log \log n)^{2},
$$

which completes the proof of 22 .

\section{Hilbert-Schmidt bounds}

This section contains general bounds on Dirac operators in terms of their defining paths.

Proposition 14 (Hilbert-Schmidt property). Let $\{\gamma(t), 0 \leq t\}$ be a measurable path in $\mathbb{H}$, $\eta_{0}, \eta_{1} \in \partial \mathbb{H}$ distinct boundary points and $z_{0} \in \mathbb{H}$. For $a \nu>0$ set $\tilde{\gamma}(t)=\gamma\left(\nu \log \left(\frac{1}{1-t}\right)\right)$. Let $z(t)$ be the point moving with speed $\alpha>0$ on the geodesic connecting $z_{0}$ to $\eta_{1}$ with $z(0)=z_{0}$. Assume that there are constants $b>0$ and $\varepsilon \in\left(0, \nu^{-1}\right)$ so that

$$
d_{\mathbb{H}}(\gamma(t), z(t)) \leq b+\varepsilon t .
$$

Then the operator

$$
\tau=\operatorname{Dir}\left(\tilde{\gamma}(t), \eta_{0}, \eta_{1}\right)
$$

is self-adjoint on the appropriate domain and $\tau^{-1}$ is Hilbert-Schmidt.

Proof. We will check that the conditions of Theorem 4 are satisfied.

If $Q$ is an isometry of $\mathbb{H}$ then $d_{\mathbb{H}}\left(z_{1}, z_{2}\right)=d_{\mathbb{H}}\left(Q z_{1}, Q z_{2}\right)$ and $d_{\eta}\left(z_{1}, z_{2}\right)=d_{Q \eta}\left(Q z_{1}, Q z_{2}\right)$. Take an isometry $Q$ for which $Q z_{0}=i$ and $Q \eta_{1}=\infty$ and denote $Q \eta_{0}$ by $q$. The geodesic 
$z(t)$ is mapped into the geodesic connecting $i$ with $\infty$ with speed $\alpha$, thus $Q z(t)=i e^{\alpha t}$. From (10) it follows that

$$
\begin{aligned}
& d_{\eta_{1}}\left(z(t), z_{0}\right)=d_{\infty}\left(i e^{\alpha t}, i\right)=-\alpha t \\
& d_{\eta_{0}}\left(z(t), z_{0}\right)=d_{q}\left(i e^{\alpha t}, q\right)=\alpha t-\log \left(1+q^{2}\right) \leq \alpha t
\end{aligned}
$$

From the triangle inequality we get

$$
\begin{aligned}
& d_{\eta_{1}}\left(\gamma(t), z_{0}\right) \leq d_{\eta_{1}}\left(z(t), z_{0}\right)+d_{\mathbb{H}}(\gamma(t), z(t)) \leq-(\alpha-\varepsilon) t+b \\
& d_{\eta_{0}}\left(\gamma(t), z_{0}\right) \leq d_{\eta_{0}}\left(z(t), z_{0}\right)+d_{\mathbb{H}}(\gamma(t), z(t)) \leq(\alpha+\varepsilon) t+b .
\end{aligned}
$$

The bounds in 11 now follow easily:

$$
\int_{0}^{1} e^{d_{\eta_{1}}\left(\tilde{\gamma}(t), z_{0}\right)} d t \leq \int_{0}^{1} e^{-(\alpha-\varepsilon) \nu \log \left(\frac{1}{1-t}\right)+b} d t=e^{b} \int_{0}^{1}(1-t)^{(\alpha-\varepsilon) \nu} d t=\frac{e^{b}}{1+(\alpha-\varepsilon) \nu}<\infty
$$

and

$$
\begin{aligned}
\int_{0}^{1} \int_{0}^{t} e^{d_{\eta_{0}}\left(\tilde{\gamma}(s), z_{0}\right)+d_{\eta_{1}}\left(\tilde{\gamma}(t), z_{0}\right)} d s d t & \leq e^{2 b} \int_{0}^{1} \int_{0}^{t}(1-s)^{-(\alpha+\varepsilon) \nu}(1-t)^{(\alpha-\varepsilon) \nu} d s d t \\
& =\frac{e^{2 b}}{2(1+(\alpha-\varepsilon) \nu)(1-\varepsilon \nu)}<\infty
\end{aligned}
$$

Recall from (13) that $r \tau$ is an integral operator with kernel $K_{\mathrm{r} \tau}$. For $0<T<1$ we denote by $\boldsymbol{r}_{T} \tau$ the integral operator with kernel $K_{\mathrm{r} \tau}(x, y) \cdot \mathbf{1}(0 \leq x \leq T, 0 \leq y \leq T)$.

Proposition 15 (Hilbert-Schmidt truncation). Let $\gamma, \eta_{0}, \eta_{1}, z_{0}, \alpha, \nu, \tilde{\gamma}, z(t)$ be as in Proposition 14, and define $\tau$ according to (26).

1. Assume that (25) holds for some $b>0, \varepsilon \in\left(0, \nu^{-1}\right)$ with $\alpha \neq \nu^{-1}-\varepsilon$. Then for any $T>0$ we have

$$
\left\|\mathrm{r} \tau-\mathrm{r}_{T} \tau\right\|_{\mathrm{HS}}^{2} \leq C(1-T)^{1+\min (\nu(\alpha-\varepsilon), 1-2 \varepsilon \nu)}
$$

with $C$ depending on $\eta_{0}, \eta_{1}, 1-\nu \varepsilon, \alpha, b$.

2. Assume that for some $0<T<1$ (25) holds for $0 \leq t \leq \nu \log \left(\frac{1}{1-T}\right)$ with some $b>0$ and $\varepsilon \in\left(0, \nu^{-1}\right)$ where $\alpha \neq \nu^{-1}-\varepsilon$. Assume further that $d_{\mathbb{H}}(\tilde{\gamma}(t), \tilde{\gamma}(T)) \leq M$ for $t \geq T$. Then we have

$$
\left\|\mathrm{r} \tau-\mathrm{r} \tau_{T}\right\|_{\mathrm{HS}}^{2} \leq C e^{2 M}(1-T)^{1+\min (\nu(\alpha-\varepsilon), 1-2 \varepsilon \nu)} .
$$

with $C$ depending on $\eta_{0}, \eta_{1}, 1-\nu \varepsilon, \alpha, b$. 
Proof. We denote the representation of $\gamma$ in the half plane by $x+i y$ and use $\tilde{x}+i \tilde{y}$ for the representation of $\tilde{\gamma}$. We represent $\eta_{0}, \eta_{1}$ with nonzero vectors $u_{0}, u_{1}$ that satisfy $u_{0}^{t} J u_{1}=1$. Recall the integral kernel of $r \tau$ from (13). From the definition of $\boldsymbol{r}_{T} \tau$ we get

$$
2\left\|\mathrm{r} \tau-\mathrm{r}_{T} \tau\right\|_{\mathrm{HS}}^{2}=\int_{T}^{1} \int_{s}^{1}|a(s)|^{2}|c(t)|^{2} d t d s+\int_{0}^{T} \int_{T}^{1}|a(s)|^{2}|c(t)|^{2} d s d t
$$

where $a(s)=X(s) u_{0}$ and $c(s)=X(s) u_{1}$ with $X=\frac{1}{\sqrt{\tilde{y}}}\left(\begin{array}{cc}1 & -\tilde{x} \\ 0 & \tilde{y}\end{array}\right)$.

From 100 one can check that if $u \in \mathbb{R}^{2}$ is a nonzero vector then $e^{d_{u}(x+i y, i)}=\frac{|X u|^{2}}{|u|^{2}}$. Using the triangle inequality we get

$$
\begin{aligned}
& |a(s)|^{2}=\left|X(s) u_{0}\right|^{2}=\left|u_{0}\right|^{2} e^{d_{\eta_{0}}(\tilde{\gamma}, i)} \leq\left|u_{0}\right|^{2} e^{d_{\eta_{0}}\left(\tilde{\gamma}, z_{0}\right)+d_{\mathbb{H}}\left(z_{0}, i\right)}, \\
& |c(s)|^{2}=\left|X(s) u_{1}\right|^{2}=\left|u_{1}\right|^{2} e^{d_{\eta_{1}}(\tilde{\gamma}, i)} \leq\left|u_{1}\right|^{2} e^{d_{\eta_{1}}\left(\tilde{\gamma}, z_{0}\right)+d_{\mathbb{H}}\left(z_{0}, i\right)} .
\end{aligned}
$$

Using now 27) and recalling $\tilde{\gamma}(t)=\gamma\left(\nu \log \left(\frac{1}{1-t}\right)\right)$ we get that if 25 holds for all $t$ then

$$
|c(t)|^{2} \leq C(1-t)^{(\alpha-\varepsilon) \nu}, \quad|a(t)|^{2} \leq C(1-t)^{-(\alpha+\varepsilon) \nu},
$$

where $C$ depends on $u_{0}, u_{1}$ and $b$. This leads to

$$
\begin{aligned}
2\left\|\mathrm{r} \tau-\mathrm{r}_{T} \tau\right\|_{\mathrm{HS}}^{2} \leq & C^{2} \int_{T}^{1} \int_{s}^{1}(1-t)^{(\alpha-\varepsilon) \nu}(1-s)^{-(\alpha+\varepsilon) \nu} d t d s \\
& \quad+C^{2} \int_{0}^{T} \int_{T}^{1}(1-t)^{(\alpha-\varepsilon) \nu}(1-s)^{-(\alpha+\varepsilon) \nu} d s d t \\
= & C^{2} \frac{(1-T)^{2-2 \varepsilon \nu}}{2(1+(\alpha-\varepsilon) \nu)(1-\varepsilon \nu)}+C^{2} \frac{(1-T)^{(\alpha-\varepsilon) \nu+1}\left((1-T)^{-\nu(\alpha+\varepsilon)+1}-1\right)}{(1+(\alpha-\varepsilon) \nu)(1-\nu(\alpha+\varepsilon))} .
\end{aligned}
$$

Considering the $\nu^{-1}-\varepsilon-\alpha>0$ and $\nu^{-1}-\varepsilon-\alpha<0$ cases separately we get (28).

Now assume that 25 holds for $0 \leq t \leq \nu \log \left(\frac{1}{1-T}\right)$, and $d_{\mathbb{H}}(\tilde{\gamma}(t), \tilde{\gamma}(T)) \leq M$ for $t \geq T$. Then for $0 \leq t \leq T$ we still have 30 , while for $t \geq T$ we can use $d_{\eta}(x, y) \leq d_{\eta}(x, z)+d_{\mathbb{H}}(y, z)$ to get

$$
|c(t)|^{2} \leq C e^{M}(1-T)^{(\alpha-\varepsilon) \nu}, \quad|a(t)|^{2} \leq C e^{M}(1-T)^{-(\alpha+\varepsilon) \nu}, \quad \text { for } T \leq t<1 .
$$

This gives

$$
\begin{aligned}
2\left\|\mathrm{r} \tau-\mathrm{r} \tau_{T}\right\|_{\mathrm{HS}}^{2} \leq & C^{2} e^{2 M} \int_{T}^{1} \int_{s}^{1}(1-T)^{-2 \varepsilon \nu} d t d s \\
& \quad+C^{2} e^{2 M} \int_{0}^{T}(1-s)^{-(\alpha+\varepsilon) \nu} d s \int_{T}^{1}(1-T)^{(\alpha-\varepsilon) \nu} d t \\
= & C^{2} e^{2 M}(1-T)^{2-2 \varepsilon \nu}+C^{2} e^{2 M} \frac{(1-T)^{(\alpha-\varepsilon) \nu+1}\left((1-T)^{-\nu(\alpha+\varepsilon)+1}-1\right)}{1-\nu(\alpha+\varepsilon)},
\end{aligned}
$$

from which 29 follows. 
Proposition 16 (Hilbert-Schmidt approximation). Let $\gamma, \eta_{0}, \eta_{1}, z_{0}, \alpha, \nu, \tilde{\gamma}, z(t)$ be as in Proposition 14, and define $\tau$ according to (26). Assume that for some $0<T<1$ (25) holds for $0 \leq t \leq \nu \log \left(\frac{1}{1-T}\right)$ for some $b>0$ and $0<\varepsilon<\min \left(\alpha, \frac{1}{2 \nu}\right)$.

Suppose that the path $\tilde{\gamma}_{1}$ is measurable and for $0 \leq t \leq \nu \log \left(\frac{1}{1-T}\right)$ we have

$$
\sinh \left(\frac{1}{2} d_{\mathbb{H}}\left(\tilde{\gamma}(t), \tilde{\gamma}_{1}(t)\right)\right)^{2} \leq \min \left(\delta(1-t)^{-1}, M\right)
$$

for some $M, \delta>0$.

Consider $\tau_{1}=\operatorname{Dir}\left(\left\{\tilde{\gamma}_{1}(t), t \geq 0\right\}, \eta_{0}, \eta_{1}\right)$, define $\boldsymbol{r}_{T} \tau$ as in Proposition 15, and $\mathbf{r}_{T} \tau_{1}$ similarly. Then

$$
\left\|r_{T} \tau-r_{T} \tau_{1}\right\|_{\mathrm{HS}}^{2} \leq C(M+1) \delta
$$

with a constant $C$ depending only on $\eta_{0}, \eta_{1}, \alpha, \nu, \varepsilon, b$.

Proof. Denote the representation of $\tilde{\gamma}_{1}$ in the half plane by $\tilde{x}_{1}+i \tilde{y}_{1}$.

The hyperbolic distance formula in the upper half plane representation gives

$$
4 \sinh \left(\frac{1}{2} d_{\mathbb{H}}\left(\tilde{\gamma}, \tilde{\gamma}_{1}\right)\right)^{2}=\frac{\left(\tilde{x}-\tilde{x}_{1}\right)^{2}}{\tilde{y} \tilde{y}_{1}}+\left(\sqrt{\frac{\tilde{y}}{\tilde{y}_{1}}}-\sqrt{\frac{\tilde{y}_{1}}{\tilde{y}}}\right)^{2}
$$

Consider $a, c$, defined as in the proof of Proposition 15 and the analogously defined $a_{1}, c_{1}$.

If $u \in \mathbb{R}^{2}$ is a nonzero vector, $X=\frac{1}{\sqrt{\tilde{y}}}\left(\begin{array}{cc}1 & -\tilde{x} \\ 0 & \tilde{y}\end{array}\right)$ and $X_{1}=\frac{1}{\sqrt{\tilde{y}_{1}}}\left(\begin{array}{cc}1 & -\tilde{x}_{1} \\ 0 & \tilde{y}_{1}\end{array}\right)$ then

$$
\frac{\left|X u-X_{1} u\right|}{|X u|}=\frac{\left|\left(I-X_{1} X^{-1}\right) X u\right|}{|X u|} \leq\left\|I-X_{1} X^{-1}\right\|_{2}^{2}
$$

An explicit computation gives

$$
\begin{aligned}
\left\|I-X_{1} X^{-1}\right\|_{2}^{2} & =\left(\frac{x-x_{1}}{\sqrt{\tilde{y} \tilde{y}_{1}}}\right)^{2}+\left(1-\sqrt{\frac{\tilde{y}_{1}}{\tilde{y}}}\right)^{2}+\left(1-\sqrt{\frac{\tilde{y}}{\tilde{y}_{1}}}\right)^{2} \\
& \leq\left(\frac{\tilde{x}-\tilde{x}_{1}}{\sqrt{\tilde{y} \tilde{y}_{1}}}\right)^{2}+\left(\sqrt{\frac{\tilde{y}}{\tilde{y}}}-\sqrt{\frac{\tilde{y}_{1}}{\tilde{y}}}\right)^{2}=4 \sinh \left(\frac{1}{2} d_{\mathbb{H}}\left(\tilde{x}+i \tilde{y}, \tilde{x}_{1}+i \tilde{y}_{1}\right)\right)^{2} .
\end{aligned}
$$

This yields

$$
\frac{\left|c-c_{1}\right|^{2}}{|c|^{2}} \leq 4 \sinh \left(\frac{1}{2} d_{\mathbb{H}}\left(\tilde{\gamma}, \tilde{\gamma}_{1}\right)\right)^{2}, \quad \frac{\left|a-a_{1}\right|^{2}}{|a|^{2}} \leq 4 \sinh \left(\frac{1}{2} d_{\mathbb{H}}\left(\tilde{\gamma}, \tilde{\gamma}_{1}\right)\right)^{2} .
$$

To compute $\left\|r_{T} \tau-r_{T} \tau_{1}\right\|_{\text {HS }}^{2}$ we need to estimate $\operatorname{tr}\left(\Delta K(s, t) \Delta K(s, t)^{t}\right)$ where $\Delta K(s, t)=$ $\frac{1}{2}\left(a(s) c(t)^{t}-a_{1}(s) c_{1}(t)^{t}\right)$. Using the Cauchy-Schwarz inequality we obtain

$$
\begin{aligned}
& 4 \operatorname{tr}\left(\Delta K(s, t) \Delta K(s, t)^{t}\right) \\
& \quad \leq 3\left(|a(s)|^{2}|| c(t)-\left.c_{1}(t)\right|^{2}+\left|a(s)-a_{1}(s)\right|^{2}|c(t)|^{2}+\left|a(s)-a_{1}(s)\right|^{2}\left|c(t)-c_{1}(t)\right|^{2}\right) .
\end{aligned}
$$


The previous estimates with (31) yield

$$
\begin{aligned}
\| r_{T} \tau & -r_{T} \tau_{1} \|_{\mathrm{HS}}^{2}=2 \int_{0}^{T} \int_{0}^{t} \operatorname{tr}\left(\Delta K(s, t) \Delta K(s, t)^{t}\right) d s d t \\
& \leq 24 \int_{0}^{T} \int_{0}^{t}|a(s)|^{2}|c(t)|^{2}\left(\sinh \left(\frac{d_{H}(s)}{2}\right)^{2}+\sinh \left(\frac{d_{H}(t)}{2}\right)^{2}+\sinh \left(\frac{d_{H}(s)}{2}\right)^{2} \sinh \left(\frac{d_{H}(t)}{2}\right)^{2}\right) d s d t \\
& \leq 24 \int_{0}^{T} \int_{0}^{t}|a(s)|^{2}|c(t)|^{2}\left(\delta(1-t)^{-1}+\delta(1-s)^{-1}+\delta^{2}(1-t)^{-1}(1-s)^{-1}\right) d s d t .
\end{aligned}
$$

Using the arguments in the proof of Proposition 15 we get that $(30)$ holds for $0 \leq t \leq T$, with a constant $C_{1}$ depending on $u_{0}, u_{1}$ and $b$. Using this together with the bound $\delta(1-t)^{-1} \leq M$ (which holds for $t \in[0, T]$ by assumption) we get

$$
\begin{aligned}
& \left\|\mathrm{r}_{T} \tau-\mathrm{r}_{T} \tau_{1}\right\|_{\mathrm{HS}}^{2} \\
& \quad \leq 24 C_{1}^{2} \int_{0}^{T} \int_{0}^{t}(1-s)^{-(\alpha+\varepsilon) \nu}(1-t)^{(\alpha-\varepsilon) \nu}\left(\delta(1-t)^{-1}+\delta(1-s)^{-1}+\delta^{2}(1-t)^{-1}(1-s)^{-1}\right) d s d t \\
& \quad \leq 24 C_{1}^{2} \delta\left(1+\frac{M}{2}\right) \int_{0}^{T} \int_{0}^{t}\left((1-s)^{-(\alpha+\varepsilon) \nu-1}(1-t)^{(\alpha-\varepsilon) \nu}+(1-s)^{-(\alpha+\varepsilon) \nu}(1-t)^{(\alpha-\varepsilon) \nu-1}\right) d s d t \\
& \quad \leq 24 C_{1}^{2} \delta(2+M) \frac{1}{(\alpha-\varepsilon) \nu(1-2 \varepsilon \nu)} .
\end{aligned}
$$

The bound 32 now follows with the choice $C=\frac{48 C_{1}^{2}}{(\alpha-\varepsilon) \nu(1-2 \varepsilon \nu)}$.

\section{Proof of Theorem 1}

We can now return to the proof of our main theorem.

Proof of Theorem 11. Let $\mathcal{B}$ be a hyperbolic Brownian motion. Set $\tilde{\mathcal{B}}(t)=\mathcal{B}\left(-\frac{4}{\beta} \log (1-t)\right)$ for $0 \leq t<1$. Set $\eta_{0}=\infty, \eta_{1}=\mathcal{B}(\infty)$ and $\operatorname{set} \operatorname{Sine}_{\beta}=\operatorname{Dir}\left(\tilde{\mathcal{B}}, \eta_{0}, \eta_{1}\right)$.

Let $U$ be a uniform random variable on $[0,1]$ independent of $\mathcal{B}$ and let $\tau_{n, k}$ be the stopping times constructed in Proposition 12 . According to the proposition the path $\tilde{\mathcal{B}}_{n}(t)=$ $\mathcal{B}\left(\tau_{n,\lceil(1-t) n]}\right), t \in[0,1)$ has the same distribution as the path in the construction of $\operatorname{Circ}_{\beta, n}$ in Theorem 8, and we may write $\operatorname{Circ}_{\beta, n}=\operatorname{Dir}\left(\tilde{\mathcal{B}}_{n}, \eta_{0}, \eta_{1}\right)$.

To prove the bound $(1)$ we set $T_{n}=1-\frac{\log ^{6} n}{n}$. Recall the definition of $\boldsymbol{r}_{T}$ from Section 4 and write

$$
\begin{aligned}
\left\|r \operatorname{Sine}_{\beta}-\operatorname{rirc}_{\beta, n}\right\|_{\mathrm{HS}}^{2} \leq & 3\left\|r_{T_{n}} \operatorname{Sine}_{\beta}-\mathrm{r}_{T_{n}} \operatorname{Circ}_{\beta, n}\right\|_{\mathrm{HS}}^{2} \\
& +3\left\|r \operatorname{Sine}_{\beta}-r_{T_{n}} \operatorname{Sine}_{\beta}\right\|_{\mathrm{HS}}^{2}+3\left\|r \operatorname{Circ}_{\beta, n}-r_{T_{n}} \operatorname{Circ}_{\beta, n}\right\|_{\mathrm{HS}}^{2} .
\end{aligned}
$$

We will use Propositions 13,15 and 16 to estimate the three terms on the right. Let $z(t)$ be the point moving with speed $1 / 2$ on the geodesic connecting $\mathcal{B}(0)$ to $\mathcal{B}(\infty)$. From Lemma 
21 of the Appendix it follows that for any $\varepsilon>0$ there is a random $b=b_{\varepsilon}$ so that a.s. for all $t \geq 0$ we have

$$
d_{\mathbb{H}}(\mathcal{B}(t), z(t)) \leq \varepsilon t+b
$$

Applying the first statement of Proposition 15 for $\operatorname{Sine}_{\beta}=\operatorname{Dir}\left(\tilde{\mathcal{B}}(t), \eta_{0}, \eta_{1}\right)$ with $\gamma=\mathcal{B}$, $\nu=\frac{4}{\beta}, \alpha=1 / 2$ and an $\varepsilon>0$ satisfying $0<\varepsilon<\min \left(\frac{1}{4}, \frac{\beta}{16}\right)$ we get

$$
\| \mathrm{r} \operatorname{Sine}_{\beta}-\mathrm{r}_{T_{n}} \text { Sine }_{\beta} \|_{\mathrm{HS}}^{2} \leq C\left(\frac{\log ^{6} n}{n}\right)^{1+\min \left(\frac{1}{\beta}, \frac{1}{2}\right)}
$$

with a random $C$ depending on $\mathcal{B}$ and $\beta$.

Recall from Proposition 13 that for $n \geq N^{*}$ we have

$$
\begin{aligned}
& d_{\mathbb{H}}\left(\tilde{\mathcal{B}}(t), \tilde{\mathcal{B}}_{n}(t)\right) \leq \frac{\log ^{3-1 / 8} n}{\sqrt{(1-t) n}} \leq 1, \quad \text { if } \quad 0 \leq t \leq T_{n}, \\
& d_{\mathbb{H}}\left(\tilde{\mathcal{B}}_{n}\left(T_{n}\right), \tilde{\mathcal{B}}_{n}(t)\right) \leq \frac{144}{\beta}(\log \log n)^{2}, \quad \text { if } \quad T_{n} \leq t<1 .
\end{aligned}
$$

Let $\mathcal{B}_{n}(t)=\tilde{\mathcal{B}}_{n}\left(1-e^{-\frac{\beta}{4} t}\right)$, then $\tilde{\mathcal{B}}_{n}(t)=\mathcal{B}_{n}\left(\frac{4}{\beta} \log \left(\frac{1}{1-t}\right)\right)$ for $0 \leq t<1$. From $(34$, , 35 and the triangle inequality we get

$$
d_{\mathbb{H}}\left(\mathcal{B}_{n}(t), z(t)\right) \leq \varepsilon t+b+1, \text { for } 0 \leq t \leq \frac{4}{\beta} \log \left(\frac{1}{1-T_{n}}\right)
$$

Recall that $\operatorname{Circ}_{\beta, n}=\operatorname{Dir}\left(\tilde{\mathcal{B}}_{n}(t), \eta_{0}, \eta_{1}\right)$. Applying the second statement of Proposition 15 with $\gamma=\mathcal{B}_{n}, \nu=\frac{4}{\beta}, \alpha=1 / 2, M=\frac{144}{\beta}(\log \log n)^{2}, T=T_{n}$ and an $\varepsilon>0$ satisfying $0<\varepsilon<\min \left(\frac{1}{4}, \frac{\beta}{16}\right)$ we get

$$
\left\|r \operatorname{Circ}_{\beta, n}-r_{T_{n}} \operatorname{Circ}_{\beta, n}\right\|_{\mathrm{HS}}^{2} \leq C e^{\frac{288}{\beta}(\log \log n)^{2}}\left(\frac{\log ^{6} n}{n}\right)^{1+\min \left(\frac{1}{\beta}, \frac{1}{2}\right)} \quad \text { if } n \geq N^{*}
$$

with a random $C$ depending on $\mathcal{B}$ and $\beta$.

Since $\sinh (x / 2)^{2} \leq x^{2}$ for $0 \leq x \leq 1$, from 35 we get

$$
\sinh \left(\frac{1}{2} d_{H}\left(\mathcal{B}(t), \mathcal{B}_{n}(t)\right)\right)^{2} \leq \frac{\log ^{6-1 / 4} n}{n}(1-t)^{-1} \leq 1, \quad \text { if } \quad 0 \leq t \leq T_{n}
$$

Hence we may use Proposition 16 with $\gamma=\mathcal{B}, \gamma_{1}=\mathcal{B}_{n}, \nu=\frac{4}{\beta}, \alpha=1 / 2, T=T_{n}$, $\delta=\frac{\log ^{6-1 / 4} n}{n}, M=1$ and $0<\varepsilon<\min \left(\frac{1}{2}, \frac{\beta}{2}\right)$ to get

$$
\left\|\mathrm{r}_{T_{n}} \operatorname{Sine}_{\beta}-\mathrm{r}_{T_{n}} \operatorname{Circ}_{\beta, n}\right\|_{\mathrm{HS}}^{2} \leq C \frac{\log ^{6-1 / 4} n}{n} \quad \text { if } n \geq N^{*}
$$


with a random $C$ depending on $\mathcal{B}$ and $\beta$. Collecting our estimates, going back to (33) and modifying the random lower bound $N^{*}$ appropriately we get

$$
\left\|r \operatorname{Sine}_{\beta}-r \operatorname{Circ}_{\beta, n}\right\|_{\mathrm{HS}}^{2} \leq \frac{\log ^{6} n}{n}, \quad \text { for } n \geq N^{*} .
$$

This completes the proof of Theorem 1 .

Before proving the three statements of Corollary 2 we state a law of large numbers for the points of Sine $_{\beta}$.

Proposition 17. Suppose that the points of Sine $_{\beta}$ are given by $\lambda_{k}, k \in \mathbb{Z}$ with $\lambda_{0}<0<\lambda_{1}$. Then with probability one we have

$$
\lim _{k \rightarrow \infty} \frac{\lambda_{k}}{k}=\lim _{k \rightarrow-\infty} \frac{\lambda_{k}}{k}=2 \pi
$$

Proof. We will show the statement for $k \rightarrow \infty$, by the symmetry of the Sine $_{\beta}$ process this is sufficient. In Holcomb and Valkó (2015) it was shown that $\frac{1}{\lambda} \#\left\{\lambda_{k}: 0 \leq \lambda_{k} \leq \lambda\right\}$ satisfies a large deviation principle as $\lambda \rightarrow \infty$ with scale $\lambda^{2}$ and rate function $\beta I(\rho)$, where $I\left(\frac{1}{2 \pi}\right)=0$ is the global minimum. From this the statement follows by a standard BorelCantelli argument.

Proof of Corollary 2. The bound (2) follows directly from (1) and the Hoffman-Wielandt inequality for compact integral operators (see e.g. Bhatia and Elsner (1994)).

From Proposition 17 we see that there is a random constant $C$ so that a.s. $\left|\lambda_{k}\right| \leq C(|k|+1)$ for all $k$. Set $a_{n}=\frac{n^{1 / 4}}{\log ^{2} n}$. From $[1]$ it follows that for large enough $n$ we have

$$
\sup _{k}\left|\lambda_{k}^{-1}-\lambda_{k, n}^{-1}\right| \leq \sqrt{\sum_{k}\left|\lambda_{k}^{-1}-\lambda_{k, n}^{-1}\right|^{2}} \leq \frac{\log ^{3} n}{n^{1 / 2}} .
$$

Let $b_{k, n}=\lambda_{k}^{-1}-\lambda_{k, n}^{-1}$, then

$$
\left(\lambda_{k, n}-\lambda_{k}\right)\left(1-b_{k, n} \lambda_{k}\right)=b_{k, n} \lambda_{k}^{2}
$$

For large enough $n$ we have

$$
\max _{|k| \leq a_{n}}\left|b_{k, n} \lambda_{k}\right| \leq \frac{\log ^{3} n}{n^{1 / 2}} C\left(a_{n}+1\right)=C\left(\frac{\log n}{n^{1 / 4}}+\frac{\log ^{3} n}{n^{1 / 2}}\right)
$$

which means that $\lim _{n \rightarrow \infty} \max _{|k| \leq a_{n}}\left|b_{k, n} \lambda_{k}\right|=0$ a.s. From $(39)$, for large enough $n$, we have

$$
\max _{|k| \leq a_{n}}\left|\lambda_{k, n}-\lambda_{k}\right| \leq 2 C^{2}\left(a_{n}+1\right)^{2} \max _{|k| \leq a_{n}}\left|b_{k, n}\right| \leq 4 C^{2} a_{n}^{2} \frac{\log ^{3} n}{n^{1 / 2}}=\frac{4 C^{2}}{\log n}
$$


This completes the proof of (3).

By Proposition 17 we may choose a random $C>0$ so that $\left|\lambda_{k}\right| \leq C \sqrt{1+k^{2}}$ for all $k$. Then we have $\frac{1}{C \sqrt{1+k^{2}}} \leq \frac{1}{\left|\lambda_{k}\right|}$ and for $n \geq N,|k| \leq n^{1 / 2-\varepsilon}$ we have

$$
\frac{1}{\left|\lambda_{k, n}\right|} \geq \frac{1}{\left|\lambda_{k}\right|}-\left|\frac{1}{\lambda_{k}}-\frac{1}{\lambda_{k, n}}\right| \geq \frac{1}{C \sqrt{1+k^{2}}}-\frac{\log ^{3} n}{\sqrt{n}} \geq \frac{1}{2 C \sqrt{1+k^{2}}}
$$

(For the last inequality one might need to change $N$.) Then for such $k$ and $n$ we get

$$
\left|\lambda_{k}-\lambda_{k, n}\right|=\left|\frac{1}{\lambda_{k}}-\frac{1}{\lambda_{k, n}}\right|\left|\lambda_{k}\right|\left|\lambda_{k, n}\right| \leq \frac{\log ^{3} n}{n^{1 / 2}} 2 C^{2}\left(1+k^{2}\right) \leq \frac{1+k^{2}}{n^{1 / 2-\varepsilon}},
$$

again by setting a large enough random lower bound on $n$.

\section{Estimating the dependence on $\beta$ in Sine $_{\beta}$}

The construction of the $\mathrm{Sine}_{\beta}$ operator provides a natural coupling of this operator for all values of $\beta$. The techniques we developed for the proof of Theorem 1 can be used to estimate how the operator Sine $_{\beta}$ (and the process Sine $_{\beta}$ ) depends on the value of $\beta$ in this coupling.

Proof of Theorem 3. We have

$$
\operatorname{Sine}_{\beta}=\operatorname{Dir}\left(\tilde{\gamma}, \eta_{0}, \eta_{1}\right), \quad \operatorname{Sine}_{\beta_{1}}=\operatorname{Dir}\left(\tilde{\gamma}_{1}, \eta_{0}, \eta_{1}\right)
$$

where $\tilde{\gamma}(t)=\mathcal{B}\left(\frac{4}{\beta} \log \left(\frac{1}{1-t}\right)\right), \tilde{\gamma}_{1}(t)=\mathcal{B}\left(\frac{4}{\beta_{1}} \log \left(\frac{1}{1-t}\right)\right), \eta_{0}=\infty$ and $\eta_{1}=\mathcal{B}(\infty)$. We will estimate $\left\|r \operatorname{Sine}_{\beta}-r \operatorname{Sine}_{\beta_{1}}\right\|_{\mathrm{HS}}$ using Propositions 15 and 16 . Note that with probability one $\eta_{0} \neq \eta_{1}$ and hence a.s. 0 is not an eigenvalue for any of the $\operatorname{Sine}_{\beta}$ operators.

Set $T=1-\delta<1$. Cutting off Sine $_{\beta}$ and Sine $_{\beta_{1}}$ at $T$ using the first statement of Proposition 15 gives

$$
\left\|\mathbf{r} \operatorname{Sine}_{\beta}-\mathbf{r}_{T} \operatorname{Sine}_{\beta}\right\|_{\mathrm{HS}}^{2}+\left\|\mathbf{r} \operatorname{Sine}_{\beta_{1}}-\mathbf{r}_{T} \operatorname{Sine}_{\beta_{1}}\right\|_{\mathrm{HS}}^{2} \leq C_{0} \delta^{1+\min (4 \kappa, 1)-\varepsilon}
$$

for a small enough $\varepsilon>0$ with a random $C_{0}$. (Recall that $\kappa \leq \beta^{-1}<\beta_{1}^{-1}$.)

To estimate $\| \mathrm{r}_{T}$ Sine $_{\beta}-\mathbf{r}_{T}$ Sine $_{\beta_{1}} \|_{\text {HS }}^{2}$ we first bound $d_{\mathbb{H}}\left(\tilde{\gamma}(t), \tilde{\gamma}_{1}(t)\right)$ in $[0, T]$. For $0<$ $t \leq T$ we have $\left(\frac{4}{\beta_{1}}-\frac{4}{\beta}\right) \log \left(\frac{1}{1-t}\right) \leq \delta \log \left(\frac{1}{\delta}\right)<1$ and by Proposition 22 (and the comment following the proposition) we get the bound

$$
d_{\mathbb{H}}\left(\mathcal { B } \left(\frac{4}{\beta} \log \left(\frac{1}{1-t}\right), \mathcal{B}\left(\frac{4}{\beta_{1}} \log \left(\frac{1}{1-t}\right)\right)^{2}<C^{2} \delta \log \left(\frac{1}{1-t}\right) \log \left(2+\frac{\frac{4}{\beta} \log \left(\frac{1}{1-t}\right)+1}{\delta \log \left(\frac{1}{1-t}\right)}\right)\right.\right.
$$

for $0 \leq t \leq T$ with a random $C$. 
The right side of (40) can be bounded by

$$
C^{2} \delta \log \left(\frac{1}{1-t}\right)\left(\log \left(\left(\frac{4}{\kappa}+2\right) \log \left(\frac{1}{\delta}\right)+1\right)+\log \left(\frac{1}{\delta \log \left(\frac{1}{1-t}\right)}\right)\right)
$$

which can further be bounded by $C^{2} c_{1}$ with a deterministic $c_{1}$ depending only $\kappa$.

Note that since $\delta<1 / 3$, we have

$$
\log \left(\frac{1}{1-t}\right) \log \left(\left(\frac{4}{\kappa}+2\right) \log \left(\frac{1}{\delta}\right)+1\right) \leq c_{2} \log \left(\frac{1}{\delta}\right)(1-t)^{-1}
$$

with a deterministic $c_{2}$ depending only on $\kappa$. Moreover, it is a straightforward calculus exercise to show that for $0<t \leq T=1-\delta$ we have

$$
\delta \log \left(\frac{1}{1-t}\right) \log \left(\frac{1}{\delta \log \left(\frac{1}{1-t}\right)}\right) \leq e^{-1 / 3} \delta \log \left(\frac{1}{\delta}\right)(1-t)^{-1} .
$$

This gives the bound

$$
d_{\mathbb{H}}\left(\tilde{\gamma}(t), \tilde{\gamma}_{1}(t)\right)^{2}<C_{1} \min \left(\delta \log \left(\frac{1}{\delta}\right)(1-t)^{-1}, 1\right)
$$

with a constant depending on $C$ and $\kappa$. We can turn this into an upper bound of the form

$$
\sinh \left(\frac{1}{2} d_{\mathbb{H}}\left(\tilde{\gamma}(t), \tilde{\gamma}_{1}(t)\right)^{2} \leq C_{2} \min \left(\delta \log \left(\frac{1}{\delta}\right)(1-t)^{-1}, 1\right),\right.
$$

from which Proposition 16 gives

$$
\| \mathrm{r}_{T} \text { Sine }_{\beta}-\mathrm{r}_{T} \text { Sine }_{\beta_{1}} \|_{\mathrm{HS}}^{2} \leq C_{3} \delta \log \left(\frac{1}{\delta}\right) .
$$

Collecting all the terms gives

$$
\left\|\mathrm{r} \mathrm{Sine}_{\beta}-\mathrm{r} \mathrm{Sine}_{\beta_{1}}\right\|_{\mathrm{HS}}^{2} \leq C \delta \log \left(\frac{1}{\delta}\right)
$$

with a random $C$. The Hoffman-Wielandt inequality finishes the proof of (5).

\section{Appendix}

In the first part of the Appendix we collect the proofs of Lemmas 9 and 11 that were used in the single step coupling of Proposition 10. In the second part we collect some estimates on the hyperbolic Brownian motion. 


\subsection{Proof of the coupling statements}

We now return to the proof of Lemma 9 , Let $\mathcal{B}$ be a hyperbolic Brownian motion and set $\zeta=\zeta_{t}=d_{\mathbb{H}}(\mathcal{B}(0), \mathcal{B}(t))$.

The circumference of a hyperbolic circle of radius $r$ is $2 \pi \sinh (r)$ and the density function of the hyperbolic BM at time $t$ and distance $r$ is given by the following formula (see e.g. Karpelevicii, Shur and Tutubalin (1959)):

$$
g(r, t)=\frac{\sqrt{2} e^{-t / 8}}{(2 \pi t)^{3 / 2}} \int_{r}^{\infty} \frac{s e^{-\frac{s^{2}}{2 t}}}{\sqrt{\cosh s-\cosh r}} d s .
$$

From this the density of $\zeta_{t}$ is

$$
p_{\zeta}(r, t)=2 \pi \sinh (r) g(r, t)=\frac{e^{-t / 8} \sinh (r)}{\sqrt{\pi} t^{3 / 2}} \int_{r}^{\infty} \frac{s e^{-\frac{s^{2}}{2 t}}}{\sqrt{\cosh s-\cosh r}} d s,
$$

and the tail of the cumulative distribution function is

$$
\begin{aligned}
1-F_{\zeta}(r) & =\int_{r}^{\infty} \sinh (u) \frac{e^{-t / 8}}{\sqrt{\pi} t^{3 / 2}} \int_{u}^{\infty} \frac{s e^{-\frac{s^{2}}{2 t}}}{\sqrt{\cosh s-\cosh u}} d s d u \\
& =\int_{r}^{\infty} \frac{e^{-t / 8} s e^{-\frac{s^{2}}{2 t}}}{\sqrt{\pi} t^{3 / 2}} \int_{r}^{s} \frac{\sinh (u)}{\sqrt{\cosh s-\cosh u}} d u d s \\
& =\int_{r}^{\infty} \frac{2 e^{-t / 8} s e^{-\frac{s^{2}}{2 t}}}{\sqrt{\pi} t^{3 / 2}} \sqrt{\cosh (s)-\cosh (r)} d s .
\end{aligned}
$$

Let $Y=Y_{\gamma}=\log \left(\frac{1+\sqrt{\xi}}{1-\sqrt{\xi}}\right)$ where $\xi$ has distribution $\operatorname{Beta}(1, \gamma)$. We record the cumulative distribution function $F_{Y}$ and the probability density function $p_{Y}$ of $Y$, which follow from direct computation of the Beta distribution:

$$
F_{Y}(r)=1-\operatorname{sech}^{2 \gamma}\left(\frac{r}{2}\right), \quad p_{Y}(r)=\gamma \sinh \left(\frac{r}{2}\right) \operatorname{sech}^{2 \gamma+1}\left(\frac{r}{2}\right) .
$$

We start with a simple estimate.

Lemma 18. For $0 \leq r \leq s$ we have

$$
\frac{1}{\sqrt{2}} \sqrt{s^{2}-r^{2}} \leq \sqrt{\cosh (s)-\cosh (r)} \leq \frac{1}{\sqrt{2}} \sqrt{s^{2}-r^{2}} \exp \left(\frac{r^{2}+s^{2}}{24}\right)
$$

Proof. The statement follows from the bound $1 \leq \frac{\sinh (x)}{x} \leq e^{x^{2} / 6}$ and

$$
\frac{2(\cosh (s)-\cosh (r))}{s^{2}-r^{2}}=\frac{\sinh ((s-r) / 2)}{(s-r) / 2} \cdot \frac{\sinh ((s+r) / 2)}{(s+r) / 2} .
$$


Applying Lemma 18 to $(42)$ and $(43)$ and computing the resulting integrals directly we get the following bounds:

$$
\begin{aligned}
p_{\zeta}(r, t) & \geq\left(1+\frac{t}{12}\right)^{-1 / 2} \frac{1}{t} e^{-\frac{r^{2}}{2 t}-\frac{r^{2}}{12}-\frac{t}{8}} \sinh (r)=: p_{-}(r, t) \\
p_{\zeta}(r, t) & \leq \frac{1}{t} e^{-\frac{r^{2}}{2 t}-\frac{t}{8}} \sinh (r)=: p_{+}(r, t) \\
1-F_{\zeta}(r) & \leq\left(1-\frac{t}{12}\right)^{-3 / 2} e^{-\frac{r^{2}}{2 t}+\frac{r^{2}}{12}-\frac{t}{8}},
\end{aligned}
$$

where the last bound is valid for $0<t<12$.

We also record the following bounds on $\log \cosh (r)$ which can be readily checked by differentiation and Taylor expansion.

$$
\begin{array}{ll}
\log \cosh (x) \leq \frac{x^{2}}{2}, & \text { for all } x \\
\log \cosh (x) \geq \frac{x^{2}}{2}-\frac{x^{4}}{12}, & \text { for } 0 \leq x \leq 1
\end{array}
$$

Proof of (a) in Lemma 9. From (44) and 49) we get

$$
1-F_{Y}(r)=\exp (-2 \gamma \log \cosh (r / 2)) \geq \exp \left(-\gamma \frac{r^{2}}{4}\right)=\exp \left(-\frac{r^{2}}{2 t}+\frac{r^{2}}{8}\right)
$$

One can check that if $0<t \leq 1$ and $t \leq r$ then

$$
\left(1-\frac{t}{12}\right)^{-3 / 2} e^{-\frac{r^{2}}{2 t}+\frac{r^{2}}{12}-\frac{t}{8}} \leq \exp \left(-\frac{r^{2}}{2 t}+\frac{r^{2}}{8}\right) .
$$

Together with 48 this proves the statement for $t \leq r$.

To prove the statement in the $0 \leq r<t$ case, we will show

$$
\int_{0}^{r} p_{\zeta}(u, t) d u \geq \int_{0}^{r} p_{Y}(u) d u \quad \text { for } 0 \leq r<t \leq 1
$$

using the lower bound (46) on $p_{\zeta}(r, t)$. We will prove that for $0<r<t \leq 1$ we have

$$
p_{-}(r, t)=\left(1+\frac{t}{12}\right)^{-1 / 2} \frac{1}{t} e^{-\frac{r^{2}}{2 t}-\frac{r^{2}}{12}-\frac{t}{8}} \sinh (r) \geq \gamma \sinh \left(\frac{r}{2}\right) \operatorname{sech}^{2 \gamma+1}\left(\frac{r}{2}\right)=p_{Y}(r) .
$$

The last inequality is equivalent to

$$
e^{-\frac{r^{2}}{2 t}-\frac{r^{2}}{12}} \geq e^{t / 8} \sqrt{1+\frac{t}{12}}\left(1-\frac{t}{4}\right) \exp \left(-\left(\frac{4}{t}+1\right) \log \cosh (r / 2)\right) .
$$

For $0 \leq t \leq 1$ we have $e^{t / 8} \sqrt{1+\frac{t}{12}}\left(1-\frac{t}{4}\right) \leq 1$. Then by the bound 50 we get

$$
e^{t / 8} \sqrt{1+\frac{t}{12}}\left(1-\frac{t}{4}\right) \exp \left(-\left(\frac{4}{t}+1\right) \log \cosh (r / 2)\right) \leq \exp \left(-\left(\frac{4}{t}+1\right)\left(\frac{r^{2}}{8}-\frac{r^{4}}{192}\right)\right) .
$$

The finish the proof of (51) we need to show that for $0<r<t \leq 1$ we have

$$
\left(\frac{4}{t}+1\right)\left(\frac{r^{2}}{8}-\frac{r^{4}}{192}\right) \geq \frac{r^{2}}{2 t}+\frac{r^{2}}{12},
$$

which follows from direct computation. 
Now we turn to the proof of the total variation bound.

Proof of (b) in Lemma 9. We need to show that $\int_{0}^{\infty}\left|p_{Y}(r)-p_{\zeta}(r, t)\right| d r \leq 3 t$.

By part (a) we have $P(\zeta>r) \leq P\left(Y_{\gamma}>r\right)$ which leads to

$$
\int_{K}^{\infty}\left|p_{Y}(r)-p_{\zeta}(r, t)\right| d r \leq 2\left(1-F_{Y}(K)\right)=2 \operatorname{sech}^{2 \gamma}\left(\frac{K}{2}\right), \quad \text { for } K>0 .
$$

Setting $K=2 \sqrt{t \log (2 / t)} \leq 2$ we get

$$
\int_{K}^{\infty}\left|p_{Y}(r)-p_{\zeta}(r, t)\right| d r \leq 2 \operatorname{sech}^{2 \gamma}\left(\frac{K}{2}\right) \leq 2 \exp \left(-2 \gamma \cdot \frac{5}{12}(K / 2)^{2}\right) \leq 2(t / 2)^{\frac{5}{4}} \leq t,
$$

where we used (50) in the second inequality.

Using the triangle inequality and the bound 46 we get

$$
\int_{0}^{K}\left|p_{Y}(r)-p_{\zeta}(r, t)\right| d r \leq \int_{0}^{K}\left|p_{Y}(r)-p_{+}(r, t)\right| d r+\int_{0}^{K}\left(p_{+}(r, t)-p_{\zeta}(r, t)\right) d r .
$$

We can bound the second integral explicitly:

$$
\begin{aligned}
\int_{0}^{K}\left(p_{+}(r, t)-p(r, t)\right) d r & \leq \int_{0}^{\infty}\left(p_{+}(r, t)-p(r, t)\right) d r=\frac{\sqrt{\frac{\pi}{2}} e^{\frac{3 t}{8}}(2 \Phi(\sqrt{t})-1)}{\sqrt{t}}-1 \\
& \leq e^{\frac{3 t}{8}}-1 \leq t / 2 .
\end{aligned}
$$

Introduce

$$
p_{0}(r, t)=\frac{2 e^{-\frac{r^{2}}{2 t}} \sinh \left(\frac{r}{2}\right)}{t}
$$

Then

$$
\frac{p_{+}}{p_{0}}=e^{-t / 8} \cosh (r / 2), \quad \frac{p_{Y}}{p_{0}}=(1-t / 4) e^{\frac{r^{2}}{2 t}} \operatorname{sech}^{\frac{4}{t}}\left(\frac{r}{2}\right) .
$$

We have, for $0 \leq t \leq 1,0 \leq r \leq 2$ :

$$
\left|\frac{p_{+}}{p_{0}}-1\right| \leq \frac{t}{8}+\frac{r^{2}}{4}
$$

Using the bounds (49) and (50) we get that for $0 \leq r \leq K<2$ we have

$$
1=\exp \left(\frac{r^{2}}{2 t}-\frac{4}{t} \frac{r^{2}}{8}\right) \leq e^{\frac{r^{2}}{2 t}} \operatorname{sech}^{\frac{4}{t}}\left(\frac{r}{2}\right) \leq \exp \left(\frac{r^{2}}{2 t}-\frac{4}{t}\left(\frac{r^{2}}{8}-\frac{r^{4}}{192}\right)\right)=e^{\frac{r^{4}}{48 t}}
$$

This leads to

$$
\left|\frac{p_{Y}}{p_{0}}-1\right| \leq \frac{t}{4}+\left|e^{\frac{r^{4}}{48 t}}-1\right| \leq \frac{t}{4}+\frac{r^{4}}{24 t}
$$

where we used that $\frac{r^{4}}{t} \leq t \log (2 / t)^{2} \leq 1$ if $t \leq 1$. Note also that $\sinh (x) \leq \frac{6}{5} x$ for $x \leq 1$. From this

$$
\int_{0}^{K}\left|p_{Y}(r)-p_{+}(r, t)\right| d r \leq \int_{0}^{K}\left(\frac{3}{8} t+\frac{1}{4} r^{2}+\frac{r^{4}}{24 t}\right) p_{0}(r) d r \leq \frac{6}{5} \int_{0}^{\infty}\left(\frac{3}{8} t+\frac{1}{4} r^{2}+\frac{r^{4}}{24 t}\right) \frac{e^{-\frac{r^{2}}{2 t}} r}{t} d r=\frac{29}{20} t .
$$


Collecting all our estimates gives

$$
\int_{0}^{\infty}\left|p_{Y}(r)-p_{\zeta}(r, t)\right| d r \leq 3 t
$$

The proof of Lemma 11 follows a standard coupling construction, we include it for completeness.

Proof of Lemma 11. Denote the distributions of $X_{1}, X_{2}$ by $\mu_{1}, \mu_{2}$, and let $\mu=\frac{1}{2}\left(\mu_{1}+\mu_{2}\right)$. Denote that density function of $X_{i}$ with respect to $\mu$ by $f_{i}$. From our assumptions it follows that

$$
\tilde{f}_{0}(x)=\frac{1}{1-\varepsilon} \min \left(f_{1}(x), f_{2}(x)\right), \quad \tilde{f}_{1}(x)=\frac{1}{\varepsilon}\left|f_{1}(x)-f_{2}(x)\right|^{+}, \quad \tilde{f}_{2}(x)=\frac{1}{\varepsilon}\left|f_{2}(x)-f_{1}(x)\right|^{+},
$$

are also density functions with respect to $\mu$, and the distributions corresponding to $\tilde{f}_{1}, \tilde{f}_{2}$ are stochastically ordered just as $X_{1}$ and $X_{2}$. Moreover, $(1-\varepsilon) \tilde{f}_{0}+\varepsilon \tilde{f}_{i}=f_{i}$ for $i=1,2$.

Recall that if $F$ is a cumulative distribution function, $F^{-1}(x)=\sup \{y: F(y)<x\}$ is its generalized inverse, and $U \sim$ Uniform $[0,1]$ then $F^{-1}(U)$ has cumulative distribution function given by $F$.

Denote the cumulative distribution function corresponding to $\tilde{f}_{i}$ by $\tilde{F}_{i}$. Let $U_{1}, U_{2}$ be independent uniform random variables on $[0,1]$ and consider the pair of random variables

$$
\left(Y_{1}, Y_{2}\right)=\mathbf{1}\left(U_{1} \leq 1-\varepsilon\right)\left(\tilde{F}_{0}^{-1}\left(U_{2}\right), \tilde{F}_{0}^{-1}\left(U_{2}\right)\right)+\mathbf{1}\left(U_{1}>1-\varepsilon\right)\left(\tilde{F}_{1}^{-1}\left(U_{2}\right), \tilde{F}_{2}^{-1}\left(U_{2}\right)\right) .
$$

In plain words: with probability $1-\varepsilon$ we generate $(Z, Z)$ where $Z$ has density $\tilde{f}_{0}$, and with probability $\varepsilon$ we generate $\left(\tilde{X}_{1}, \tilde{X}_{2}\right)$ where $\tilde{X}_{i}$ has density $\tilde{f}_{i}$ and $\tilde{X}_{1} \leq \tilde{X}_{2}$ a.s. Then $Y_{1} \leq Y_{2}$ a.s., $P\left(Y_{1}=Y_{2}\right)=1-\varepsilon$ and $Y_{i}$ has the same distribution as $X_{i}$. Consider the regular conditional distribution of $Y_{2}$ given $Y_{1}$, and let $g(x, u)$ be the generalized inverse of the conditional cumulative distribution function of $Y_{2}$ given $Y_{1}=x$. Then $\left(X_{1}, g\left(X_{1}, U\right)\right)$ has the same joint distribution as $\left(Y_{1}, Y_{2}\right)$, and thus $g$ satisfies the requirements of the lemma.

\subsection{Hyperbolic Brownian motion estimates}

The first two lemmas give estimates on the behavior of the process $d_{\mathbb{H}}(\mathcal{B}(0), \mathcal{B}(t))$ where $\mathcal{B}$ is a (standard) hyperbolic Brownian motion.

Lemma 19. There is a coupling of a hyperbolic Brownian motion $\mathcal{B}$, a one dimensional standard Brownian motion $B$ and a 2 dimensional standard Brownian motion $W$ so that almost surely for all $t \geq 0$ we have

$$
|B(t)| \leq d_{\mathbb{H}}(\mathcal{B}(0), \mathcal{B}(t)) \leq|W(t)|+t / 2 .
$$


Proof. The process $q_{t}=d_{\mathbb{H}}(0, \mathcal{B}(t))$ satisfies the SDE

$$
d q=d b+\frac{\operatorname{coth} q}{2} d t, \quad q(0)=0
$$

where $b$ is a standard Brownian motion.

Consider the following diffusions with the same driving Brownian motion $b$ as in $q$.

$$
d q_{1}=d b+\frac{1}{2 q_{1}} d t, \quad d q_{2}=d b+\left(\frac{1}{2 q_{2}}+\frac{1}{2}\right) d t, \quad q_{1}(0)=q_{2}(0)=1 .
$$

Since $\frac{1}{x} \leq \operatorname{coth} x \leq \frac{1}{x}+1$ for $x>0$, we have a.s. $q_{1} \leq q \leq q_{2}$. The process $q_{1}$ is a 2 dimensional Bessel process, it can be written as the absolute value of a 2 dimensional Brownian motion $W=\left(B, B^{\prime}\right)$, which is at least as large as the absolute value of its first coordinate $|B|$.

For the upper bound note that we have $q_{1} \leq q_{2}$, and taking the difference of the SDEs for $q_{2}$ and $q_{1}$ we get

$$
\left(q_{2}-q_{1}\right)^{\prime}=\frac{1}{2 q_{2}}-\frac{1}{2 q_{1}}+\frac{1}{2} \leq \frac{1}{2} .
$$

Thus $q(t) \leq q_{2}(t) \leq q_{1}(t)+t / 2=|W(t)|+t / 2$, which finishes the proof.

Lemma 20. Let $\mathcal{B}$ be a standard hyperbolic Brownian motion. Then for $t>0, a>0$ we have

$$
P\left(\max _{0 \leq s \leq t} d_{\mathbb{H}}(\mathcal{B}(0), \mathcal{B}(s)) \leq a\right) \leq \frac{4}{\pi} e^{-\frac{\pi^{2} t}{8 a^{2}}}
$$

If $0<t \leq a$ then

$$
P\left(\max _{0 \leq s \leq t} d_{\mathbb{H}}(\mathcal{B}(0), \mathcal{B}(s)) \geq a\right) \leq \frac{16 \sqrt{t}}{a \sqrt{\pi}} e^{-\frac{a^{2}}{16 t}} .
$$

Proof. By Lemma 19 the process $q_{t}=d_{\mathbb{H}}(\mathcal{B}(0), \mathcal{B}(t))$ stochastically dominates $|B(t)|$ where $B$ is a standard Brownian motion. Then

$$
P\left(\max _{0 \leq s \leq t} d_{\mathbb{H}}(\mathcal{B}(0), \mathcal{B}(s)) \leq a\right) \leq P\left(\max _{0 \leq s \leq t}|B(s)| \leq a\right) \leq \frac{4}{\pi} e^{-\frac{\pi^{2} t}{8 a^{2}}}
$$

which proves (52). The last step follows from the following identity (see e.g. Mörters and Peres (2010)):

$$
P\left(\max _{0 \leq s \leq t}|B(s)| \leq u\right)=\frac{4}{\pi} \sum_{k=0}^{\infty} \frac{(-1)^{k}}{2 k+1} e^{-\frac{(2 k+1)^{2} \pi^{2} t}{8 u^{2}}} .
$$

From Lemma 19 it follows that $q_{t}=d_{\mathbb{H}}(\mathcal{B}(0), \mathcal{B}(t))$ is stochastically dominated by the process $|W(t)|+t / 2$ where $W$ is a 2 dimensional standard Brownian motion. Thus

$$
\begin{aligned}
P\left(\max _{0 \leq s \leq t} d_{\mathbb{H}}(\mathcal{B}(0), \mathcal{B}(s)) \geq a\right) & \leq P\left(\max _{0 \leq s \leq t}(|W(s)|+s / 2) \geq a\right) \leq P\left(\max _{0 \leq s \leq t}|W(s)| \geq a-t / 2\right) \\
& \leq 4 P\left(\max _{0 \leq s \leq t} B(s) \geq \frac{1}{\sqrt{2}}(a-t / 2)\right)=4 P\left(|B(t)| \geq \frac{1}{\sqrt{2}}(a-t / 2)\right) \\
& \leq \frac{8 \sqrt{t}}{(a-t / 2) \sqrt{\pi}} e^{-\frac{(a-t / 2)^{2}}{4 t}} \leq \frac{16 \sqrt{t}}{a \sqrt{\pi}} e^{-\frac{a^{2}}{16 t}} .
\end{aligned}
$$


In the second line we compared $W$ to a one dimensional standard Brownian motion $B$, then used the reflection principle. Finally we used the well known tail bound for the normal distribution and $t \leq a$.

The next lemma shows that $\mathcal{B}$ approaches its limit point with speed $1 / 2$.

Lemma 21. Let $\mathcal{B}$ be a hyperbolic Brownian motion and let $z(t)$ be the point moving with speed $1 / 2$ on the geodesic connecting $\mathcal{B}(0)$ to $\mathcal{B}(\infty)=\lim _{t \rightarrow \infty} \mathcal{B}(t)$. Then almost surely

$$
\lim _{t \rightarrow \infty} \frac{1}{t} d_{\mathbb{H}}(\mathcal{B}(t), z(t))=0
$$

Proof. Consider the half plane representation of $\mathbb{H}$ where $\mathcal{B}(0)=i$ and $\mathcal{B}(\infty)=\infty$, and denote the representation of $\mathcal{B}$ by $x+i y$. Then $x+i y$ is a hyperbolic Brownian motion conditioned to hit $\infty$, in particular it satisfies

$$
d y=y\left(d B_{1}+d t\right), \quad d x=y d B_{2}, \quad y(0)=1, \quad x(0)=0
$$

where $B_{1}, B_{2}$ are independent standard Brownian motions. (See e.g. Valkó and Virág (2017).) The geodesic connecting $i$ and $\infty$ is $i e^{t}, t \geq 0$, and we have $z(t)=i e^{t / 2}$. By the triangle inequality

$$
\begin{aligned}
d_{\mathbb{H}}(\mathcal{B}(t), z(t)) & \leq d_{\mathbb{H}}(x+i y, i y)+d_{\mathbb{H}}(i y, z(t)) \\
& \leq \operatorname{arccosh}\left(1+\frac{x^{2}}{2 y^{2}}\right)+\left|\log \left(y e^{-t / 2}\right)\right| \leq \log \left(2+\frac{x^{2}}{y^{2}}\right)+\left|\log \left(y e^{-t / 2}\right)\right|
\end{aligned}
$$

We can explicitly solve for $y$ and $x$ from the SDE: $y(t)=e^{B_{1}(t)+t / 2}, x(t)=\int_{0}^{t} e^{B_{1}(s)+s / 2} d s$. Since $\frac{1}{t}\left|\log \left(y e^{-t / 2}\right)\right|=\frac{\left|B_{1}(t)\right|}{t}$ converges to 0 a.s., for any $\delta>0$ we have

$$
C_{\delta}^{-1} e^{(1 / 2-\delta) t} \leq y(t) \leq C_{\delta} e^{(1 / 2+\delta) t}
$$

with a random $C_{\delta}$.

For the term $\log \left(2+\frac{x^{2}}{y^{2}}\right)$ we start with the observation that there is a standard Brownian motion $W$ so that $x(t)=W\left(\int_{0}^{t} y(s)^{2} d s\right)$. Since $\lim _{t \rightarrow \infty} \int_{0}^{t} y(s)^{2} d s=\infty$ by 54 , the iterated logarithm theorem implies that for any $\varepsilon>0$ we have

$$
\lim _{t \rightarrow \infty} \frac{x(t)}{\left(\int_{0}^{t} y(s)^{2} d s\right)^{1 / 2+\varepsilon}}=0 .
$$

Using (54) we get the following estimate for fixed $\varepsilon, \delta>0$ :

$$
\frac{\left(\int_{0}^{t} y(s)^{2} d s\right)^{1+2 \varepsilon}}{y(t)^{2}} \leq C^{\prime} e^{t\{(1+2 \delta)(1+2 \varepsilon)-(1-2 \delta)\}}=C^{\prime} e^{(4 \delta \varepsilon+4 \delta+2 \varepsilon) t} .
$$

This shows that $\lim _{t \rightarrow \infty} \frac{1}{t} \log \left(2+\frac{x(t)^{2}}{y(t)^{2}}\right)=0$ a.s. and completes the proof of the lemma. 
The next statement gives an estimate on the modulus of continuity of the hyperbolic Brownian motion. The proof follows that of the analogous statement for standard Brownian motion. We include it for completeness. The constants have not been optimized.

Proposition 22. Let $\mathcal{B}$ be a hyperbolic Brownian motion. Then there is a random constant $0<h_{0} \leq 1$ so that a.s.

$$
d_{\mathbb{H}}(\mathcal{B}(s), \mathcal{B}(s+h)) \leq 20 \sqrt{h \log \left(2+\frac{s+1}{h}\right)}
$$

for all $0<h \leq h_{0}$ and $0 \leq s$

Note that the proof below also shows that there is a random constant $C$ so that (55) holds for all $0<h \leq 1$ with $C$ in place of 20 .

Proof. Let $I_{m, n}=\left[m 2^{-n},(m+1) 2^{-n}\right]$ and $\Delta_{m, n}=\max _{t \in I_{m, n}} d_{\mathbb{H}}\left(\mathcal{B}\left(m 2^{-n}\right), \mathcal{B}(t)\right)$ for $m, n \geq 0$. If $2^{-n / 2}<u$ then by Lemma 20 we have

$$
P\left(\Delta_{m, n} \geq 2^{-n / 2} u\right)=P\left(\max _{0 \leq t \leq 2^{-n}} d_{\mathbb{H}}(\mathcal{B}(0), \mathcal{B}(t)) \geq 2^{-n / 2} u\right) \leq \frac{16}{u \sqrt{\pi}} e^{-\frac{u^{2}}{16}}
$$

Thus for $n \geq 0, m \geq 0$ we get

$$
P\left(\Delta_{m, n} \geq \frac{9}{2} \cdot 2^{-n / 2} \sqrt{\log \left(2^{n}+m+1\right)}\right) \leq \frac{3\left(2^{n}+m+1\right)^{-5 / 4}}{\sqrt{\log \left(2^{n}+m+1\right)}} .
$$

We have $\sum_{n=0}^{\infty} \sum_{m=0}^{\infty}\left(2^{n}+m+1\right)^{-5 / 4}<\infty$. By the Borel-Cantelli lemma there is a random $N_{0} \geq 1$ so that if $n \geq N_{0}$ then

$$
\Delta_{m, n} \leq \frac{9}{2} \sqrt{2^{-n} \log \left(2^{n}+m+1\right)} .
$$

We will show that 55 holds with $0<h \leq h_{0}=2^{-N_{0}}$ and $0 \leq s$. Let $m, n$ be nonnegative integers with $2^{-n-1}<h \leq 2^{-n}$ and $m 2^{-n} \leq s \leq(m+1) 2^{-n}$. Then we have $n \geq N_{0}$, and using the triangle inequality and (56) we get

$$
\begin{aligned}
d_{\mathbb{H}}(\mathcal{B}(s), \mathcal{B}(s+h)) & \leq 2 \Delta_{m, n}+\Delta_{m+1, n} \leq 3 \cdot \frac{9}{2} \sqrt{\left.2^{-n} \log \left(2^{n}+m+2\right)\right)} \\
& \leq 20 \sqrt{h \log \left(2+\frac{s+1}{h}\right)}
\end{aligned}
$$

which finishes the proof.

Acknowledgements. The first author was partially supported by the NSF CAREER award DMS-1053280, the NSF award DMS-1712551 and the Simons Foundation. The second author was supported by the Canada Research Chair program the NSERC Discovery Accelerator grant, the MTA Momentum Random Spectra research group, and the ERC consolidated grant 648017 (Abért). 


\section{References}

Anderson, G., Guionnet, A. and Zeitouni, O. (2009). Introduction to random matrices, Cambridge University Press.

Bhatia, R. and Elsner, L. (1994). The Hoffman-Wielandt inequality in infinite dimensions, Proceedings of the Indian Academy of Sciences - Mathematical Sciences 104(3): 483-494.

Davies, E. B. and Mandouvalos, N. (1988). Heat Kernel Bounds on Hyperbolic Space and Kleinian Groups, Proceedings of the London Mathematical Society s3-57(1): 182-208.

Dumitriu, I. and Edelman, A. (2002). Matrix models for beta ensembles, J. Math. Phys. 43(11): 5830-5847.

Edelman, A. and Sutton, B. D. (2007). From random matrices to stochastic operators, J. Stat. Phys. 127(6): 1121-1165.

Forrester, P. J. (2010). Log-gases and random matrices, Vol. 34 of London Mathematical Society Monographs Series, Princeton University Press, Princeton, NJ.

Holcomb, D. and Valkó, B. (2015). Large deviations for the $\mathrm{Sine}_{\beta}$ and $\mathrm{Sch}_{\tau}$ processes, Probability Theory and Related Fields 163(1-2): 339-378.

Karpelevicii, F. I., Shur, M. G. and Tutubalin, V. N. (1959). Limit theorems for the compositions of distributions in the Lobachevsky plane and space, Theory of Probability and Its Applications 4(4): 399-402.

Killip, R. and Stoiciu, M. (2009). Eigenvalue statistics for CMV matrices: from Poisson to clock via random matrix ensembles, Duke Math. J. 146(3): 361-399.

Maples, K., Najnudel, J. and Nikeghbali, A. (2013). Limit operators for circular ensembles, arXiv preprint, arXiv:1304.3757.

Meckes, E. S. and Meckes, M. W. (2016). Self-similarity in the circular unitary ensemble, Discrete Anal. pp. Paper No. 9, 14.

Mehta, M. L. (2004). Random matrices, Vol. 142 of Pure and Applied Mathematics (Amsterdam), third edn, Elsevier/Academic Press, Amsterdam.

Mörters, P. and Peres, Y. (2010). Brownian motion, Vol. 30, Cambridge University Press.

Nakano, F. (2014). Level statistics for one-dimensional Schrödinger operators and Gaussian beta ensemble, Journal of Statistical Physics 156(1): 66-93. 
Ramírez, J. A. and Rider, B. (2009). Diffusion at the random matrix hard edge, Comm. Math. Phys. 288(3): 887-906.

Ramírez, J. A., Rider, B. and Virág, B. (2011). Beta ensembles, stochastic Airy spectrum, and a diffusion, J. Amer. Math. Soc. 24(4): 919-944.

Valkó, B. and Virág, B. (2009). Continuum limits of random matrices and the Brownian carousel, Inventiones Math. 177: 463-508.

Valkó, B. and Virág, B. (2017). The Sine $\beta$ operator, Inventiones mathematicae 209(1): 275327.

Wigner, E. P. (1951). On a class of analytic functions from the quantum theory of collisions, Ann. of Math. (2) 53: 36-67.

Benedek Valkó

Department of Mathematics

University of Wisconsin - Madison

Madison, WI 53706, USA

valko@math. wisc.edu

Bálint Virág

Departments of Mathematics and Statistics

University of Toronto

Toronto ON M5S 2E4, Canada

balint@math.toronto .edu

and

Alfréd Rényi Institute of Mathematics

Hungarian Academy of Sciences

Reáltanoda utca 13-15,

H-1053, Budapest, Hungary 